\title{
Stability results for partial fractional differential equations with noninstantaneous impulses
}

\author{
Saïd Abbas' ${ }^{1}$, Mouffak Benchohra ${ }^{2,3}$, Ahmed Alsaedi $^{3 *}$ and Yong Zhou ${ }^{4,3}$
}

"Correspondence:

aalsaedi@hotmail.com

${ }^{3}$ Nonlinear Analysis and Applied

Mathematics (NAAM) Research

Group, Faculty of Science, King

Abdulaziz University, P.O. Box 80203,

Jeddah, 21589, Saudi Arabia

Full list of author information is

available at the end of the article

\begin{abstract}
In this article, we investigate some uniqueness and Ulam's type stability concepts for the Darboux problem of partial functional differential equations with noninstantaneous impulses and delay in Banach spaces. The main techniques rely on fractional calculus, integral equations and inequalities. Two examples are also provided to illustrate our results.

MSC: 26A33; 34K37; 34K45

Keywords: fractional differential equation; Riemann-Liouville integral; Caputo derivative; Darboux problem; noninstantaneous impulses; Ulam-Hyers-Rassias stability
\end{abstract}

\section{Introduction}

The fractional calculus deals with extensions of derivatives and integrals to noninteger orders. It represents a powerful tool in applied mathematics to study a myriad of problems from different fields of science and engineering, with many break-through results found in mathematical physics, finance, hydrology, biophysics, thermodynamics, control theory, statistical mechanics, astrophysics, cosmology, and bioengineering. There has been a significant development in ordinary and partial fractional differential equations in recent years; see the monographs of Abbas et al. [1], Kilbas et al. [2], Miller and Ross [3], Zhou $[4,5]$, the papers [6-25], and the references therein.

In [9], Abbas et al. studied some existence, uniqueness and stability results for functional partial impulsive differential equations. In [26], Wang et al. studied the stability of firstorder impulsive evolution equations.

In pharmacotherapy, the above instantaneous impulses cannot describe the certain dynamics of evolution processes. For example, one considers the hemodynamic equilibrium of a person, the introduction of the drugs in the bloodstream and the consequent absorption for the body are gradual and continuous process. From the viewpoint of general theories, Hernández and O'Regan [27] initially offered to study a new class of abstract semilinear impulsive differential equations with noninstantaneous impulses in a $P C$-normed Banach space. Meanwhile, in $[27,28]$ the authors continue to study other new classes of differential equations with noninstantaneous impulses.

(c) The Author(s) 2017. This article is distributed under the terms of the Creative Commons Attribution 4.0 International License (http://creativecommons.org/licenses/by/4.0/), which permits unrestricted use, distribution, and reproduction in any medium, provided you give appropriate credit to the original author(s) and the source, provide a link to the Creative Commons license, and indicate if changes were made. 
However, Ulam-Hyers-Rassias stability of fractional differential equations with this kind of impulses has not been studied. Motivated by recent work [29, 30], we investigate the uniqueness and Ulam-Hyers-Rassias stability of the following partial fractional differential equations with noninstantaneous impulses and finite delay:

$$
\left\{\begin{array}{l}
{ }^{c} D_{\theta_{k}}^{r} u(t, x)=f\left(t, x, u_{(t, x)}\right) ; \quad \text { if }(t, x) \in I_{k}, k=0, \ldots, m, \\
u(t, x)=g_{k}(t, x, u(t, x)) ; \quad \text { if }(t, x) \in J_{k}, k=1, \ldots, m, \\
u(t, x)=\phi(t, x) ; \quad \text { if }(t, x) \in \tilde{J}:=[-\alpha, a] \times[-\beta, b] \backslash(0, a] \times(0, b], \\
u(t, 0)=\varphi(t) ; \quad t \in[0, a], \\
u(0, x)=\psi(x) ; \quad x \in[0, b], \\
\varphi(0)=\psi(0),
\end{array}\right.
$$

where $I_{0}=\left[0, t_{1}\right] \times[0, b], I_{k}:=\left(s_{k}, t_{k+1}\right] \times[0, b], J_{k}:=\left(t_{k}, s_{k}\right] \times[0, b] ; k=1, \ldots, m, a, b, \alpha, \beta>$ $0, \theta_{k}=\left(s_{k}, 0\right) ; k=0, \ldots, m,{ }^{c} D_{\theta_{k}}^{r}$ is the fractional Caputo derivative of order $r=\left(r_{1}, r_{2}\right) \in$ $(0,1] \times(0,1], 0=s_{0}<t_{1} \leq s_{1} \leq t_{2} \leq \cdots \leq s_{m-1} \leq t_{m} \leq s_{m} \leq t_{m+1}=a, f: I_{k} \times \mathcal{C} \rightarrow E ; k=$ $0, \ldots, m, g_{k}: J_{k} \times E \rightarrow E ; k=1, \ldots, m, \phi: \tilde{J} \rightarrow E$ are given continuous functions, $\varphi:[0, a] \rightarrow$ $E$ and $\psi:[0, b] \rightarrow E$ are given absolutely continuous functions with $\varphi(t)=\Phi(t, 0), \psi(x)=$ $\Phi(0, x)$ for each $(t, x) \in J:=[0, a] \times[0, b], E$ is a complete Banach space, and $\mathcal{C}$ is the Banach space defined by

$$
\begin{aligned}
\mathcal{C}= & C_{(\alpha, \beta)} \\
= & \{u:[-\alpha, 0] \times[-\beta, 0] \rightarrow E: \text { continuous } \\
& \text { and there exist } \tau_{k} \in(-\alpha, 0) \text { with } u\left(\tau_{k}^{-}, \tilde{x}\right) \text { and } u\left(\tau_{k}^{+}, \tilde{x}\right) ; k=1, \ldots, m, \\
& \text { exist for any } \left.\tilde{x} \in[-\beta, 0] \text { with } u\left(\tau_{k}^{-}, \tilde{x}\right)=u\left(\tau_{k}, \tilde{x}\right)\right\},
\end{aligned}
$$

with the norm

$$
\|u\|_{\mathcal{C}}=\sup _{(t, x) \in[-\alpha, 0] \times[-\beta, 0]}\|u(t, x)\|_{E^{*}}
$$

We denote by $u_{(t, x)}$ the element of $\mathcal{C}$ defined by

$$
u_{(t, x)}(\tau, \xi)=u(t+\tau, x+\xi) ; \quad(\tau, \xi) \in[-\alpha, 0] \times[-\beta, 0]
$$

here $u_{(t, x)}(\cdot, \cdot)$ represents the history of the state from time $t-\alpha$ up to the present time $t$ and from time $x-\beta$ up to the present time $x$.

Next, we consider the following partial fractional differential equations with noninstantaneous impulses and infinite delay:

$$
\left\{\begin{array}{l}
{ }^{c} D_{\theta_{k}}^{r} u(t, x)=f\left(t, x, u_{(t, x)}\right) ; \quad \text { if }(t, x) \in I_{k}, k=0, \ldots, m, \\
u(t, x)=g_{k}(t, x, u(t, x)) ; \quad \text { if }(t, x) \in J_{k}, k=1, \ldots, m, \\
u(t, x)=\phi(t, x) \quad \text { if }(t, x) \in \tilde{J}^{\prime}:=(-\infty, a] \times(-\infty, b] \backslash(0, a] \times(0, b], \\
u(t, 0)=\varphi(t) ; \quad t \in[0, a], \\
u(0, x)=\psi(x) ; \quad x \in[0, b], \\
\varphi(0)=\psi(0),
\end{array}\right.
$$


where $J, \varphi, \psi$ are as in problem (1), $f: I_{k} \times \mathcal{B} \rightarrow E ; k=0, \ldots, m, g_{k}: J_{k} \times E \rightarrow E ; k=1, \ldots, m$, $\phi: \tilde{J}^{\prime} \rightarrow E$ are given continuous functions and $\mathcal{B}$ is called a phase space that will be specified in Section 4.

\section{Preliminaries}

In this section, we introduce notations, definitions, and preliminary facts which are used throughout this paper. Denote $L^{1}(J)$ the space of Bochner-integrable functions $u: J \rightarrow E$ with the norm

$$
\|u\|_{L^{1}}=\int_{0}^{a} \int_{0}^{b}\|u(t, x)\|_{E} d x d t
$$

where $\|\cdot\|_{E}$ denotes a suitable complete norm on $E$. As usual, by $\mathrm{AC}(J)$ we denote the space of absolutely continuous functions from $J$ into $E$, and $C(J)$ is the Banach space of all continuous functions from $J$ into $E$ with the norm $\|\cdot\|_{\infty}$ defined by

$$
\|u\|_{\infty}=\sup _{(t, x) \in J}\|u(t, x)\|_{E}
$$

Let $\theta=(0,0), r_{1}, r_{2}>0$ and $r=\left(r_{1}, r_{2}\right)$. For $u \in L^{1}(J)$, the expression

$$
\left(I_{\theta}^{r} u\right)(t, x)=\frac{1}{\Gamma\left(r_{1}\right) \Gamma\left(r_{2}\right)} \int_{0}^{t} \int_{0}^{x}(t-\tau)^{r_{1}-1}(x-\xi)^{r_{2}-1} u(\tau, \xi) d \xi d \tau
$$

is called the left-sided mixed Riemann-Liouville integral of order $r$, where $\Gamma(\cdot)$ is the (Euler) Gamma function defined by $\Gamma(\varsigma)=\int_{0}^{\infty} t^{5-1} e^{-t} d t ; \varsigma>0$.

In particular,

$$
\left(I_{\theta}^{\theta} u\right)(t, x)=u(t, x), \quad\left(I_{\theta}^{\sigma} u\right)(t, x)=\int_{0}^{t} \int_{0}^{x} u(\tau, \xi) d \xi d \tau ; \quad \text { for almost all }(t, x) \in J,
$$

where $\sigma=(1,1)$. For instance, $I_{\theta}^{r} u$ exists for all $r_{1}, r_{2} \in(0, \infty)$, when $u \in L^{1}(J)$. Note also that when $u \in C(J)$, then $\left(I_{\theta}^{r} u\right) \in C(J)$, moreover,

$$
\left(I_{\theta}^{r} u\right)(t, 0)=\left(I_{\theta}^{r} u\right)(0, x)=0 ; \quad t \in[0, a], x \in[0, b]
$$

Example 2.1 Let $\lambda, \omega \in(-1,0) \cup(0, \infty), r=\left(r_{1}, r_{2}\right), r_{1}, r_{2} \in(0, \infty)$ and $h(t, x)=t^{\lambda} x^{\omega}$; $(t, x) \in J$. We have $h \in L^{1}(J)$, and we get

$$
\left(I_{\theta}^{r} h\right)(t, x)=\frac{\Gamma(1+\lambda) \Gamma(1+\omega)}{\Gamma\left(1+\lambda+r_{1}\right) \Gamma\left(1+\omega+r_{2}\right)} t^{\lambda+r_{1}} x^{\omega+r_{2}} ; \quad \text { for almost all }(t, x) \in J .
$$

By $1-r$ we mean $\left(1-r_{1}, 1-r_{2}\right) \in[0,1) \times[0,1)$. Denote by $D_{t x}^{2}:=\frac{\partial^{2}}{\partial t \partial x}$ the mixed secondorder partial derivative.

Definition 2.2 ([17]) Let $r \in(0,1] \times(0,1]$ and $u \in L^{1}(J)$. The Caputo fractional-order derivative of order $r$ of $u$ is defined by the expression

$$
{ }^{c} D_{\theta}^{r} u(t, x)=\left(I_{\theta}^{1-r} D_{t x}^{2} u\right)(t, x)=\frac{1}{\Gamma\left(1-r_{1}\right) \Gamma\left(1-r_{2}\right)} \int_{0}^{t} \int_{0}^{x} \frac{D_{\tau \xi}^{2} u(\tau, \xi)}{(t-\tau)^{r_{1}}(x-\xi)^{r_{2}}} d \xi d \tau .
$$


The case $\sigma=(1,1)$ is included and we have

$$
\left({ }^{c} D_{\theta}^{\sigma} u\right)(t, x)=\left(D_{t x}^{2} u\right)(t, x) ; \quad \text { for almost all }(t, x) \in J .
$$

Example 2.3 Let $\lambda, \omega \in(-1,0) \cup(0, \infty)$ and $r=\left(r_{1}, r_{2}\right) \in(0,1] \times(0,1]$, then

$$
{ }^{c} D_{\theta}^{r} t^{\lambda} x^{\omega}=\frac{\Gamma(1+\lambda) \Gamma(1+\omega)}{\Gamma\left(1+\lambda-r_{1}\right) \Gamma\left(1+\omega-r_{2}\right)} t^{\lambda-r_{1}} x^{\omega-r_{2}} ; \quad \text { for almost all }(t, x) \in J .
$$

Let $a_{1} \in[0, a], z^{+}=\left(a_{1}, 0\right) \in J, J_{z}=\left(a_{1}, a\right] \times[0, b], r_{1}, r_{2}>0$ and $r=\left(r_{1}, r_{2}\right)$. For $u \in L^{1}\left(J_{z}\right)$, the expression

$$
\left(I_{z^{+}}^{r} u\right)(t, x)=\frac{1}{\Gamma\left(r_{1}\right) \Gamma\left(r_{2}\right)} \int_{a_{1}^{+}}^{t} \int_{0}^{x}(t-\tau)^{r_{1}-1}(x-\xi)^{r_{2}-1} u(\tau, \xi) d \xi d \tau
$$

is called the left-sided mixed Riemann-Liouville integral of order $r$ of $u$.

Definition 2.4 ([17]) For $u \in L^{1}\left(J_{z}\right)$ where $D_{t x}^{2} u$ is Bochner integrable on $J_{z}$, the Caputo fractional-order derivative of order $r$ of $u$ is defined by the expression

$$
\left({ }^{c} D_{z^{+}}^{r} u\right)(t, x)=\left(I_{z^{+}}^{1-r} D_{t x}^{2} u\right)(t, x)
$$

Now, we consider the Ulam stability for our problems. Let $\epsilon>0, \Psi \geq 0$ and $\Phi: J \rightarrow$ $[0, \infty)$ be a continuous function. We consider the following inequalities:

$$
\begin{aligned}
& \left\{\begin{array}{l}
\left\|{ }^{c} D_{\theta_{k}}^{r} u(t, x)-f\left(t, x, u_{(t, x)}\right)\right\|_{E} \leq \epsilon ; \quad \text { if }(t, x) \in I_{k}, k=0, \ldots, m, \\
\left\|u(t, x)-g_{k}(t, x, u(t, x))\right\|_{E} \leq \epsilon ; \quad \text { if }(t, x) \in J_{k}, k=1, \ldots, m,
\end{array}\right. \\
& \left\{\begin{array}{l}
\left\|{ }^{c} D_{\theta_{k}}^{r} u(t, x)-f\left(t, x, u_{(t, x)}\right)\right\|_{E} \leq \Phi(t, x) ; \quad \text { if }(t, x) \in I_{k}, k=0, \ldots, m, \\
\left\|u(t, x)-g_{k}(t, x, u(t, x))\right\|_{E} \leq \Psi ; \quad \text { if }(t, x) \in J_{k}, k=1, \ldots, m,
\end{array}\right. \\
& \left\{\begin{array}{l}
\left\|{ }^{c} D_{\theta_{k}}^{r} u(t, x)-f\left(t, x, u_{(t, x)}\right)\right\|_{E} \leq \epsilon \Phi(t, x) ; \quad \text { if }(t, x) \in I_{k}, k=0, \ldots, m, \\
\left\|u(t, x)-g_{k}(t, x, u(t, x))\right\|_{E} \leq \epsilon \Psi ; \quad \text { if }(t, x) \in J_{k}, k=1, \ldots, m .
\end{array}\right.
\end{aligned}
$$

Definition 2.5 ( $[9,29])$ Problem $(1)$ is Ulam-Hyers stable if there exists a real number $c_{f, g_{k}}>0$ such that for each $\epsilon>0$ and for each solution $u \in P C$ of the inequality (3) there exists a solution $v \in P C$ of problem (1) with

$$
\|u(t, x)-v(t, x)\|_{E} \leq \epsilon c_{f, g_{k}} ; \quad(t, x) \in J
$$

Definition 2.6 $([9,29])$ Problem (1) is generalized Ulam-Hyers stable if there exists $c_{f, g_{k}}$ : $C([0, \infty),[0, \infty))$ with $c_{f, g_{k}}(0)=0$ such that for each $\epsilon>0$ and for each solution $u \in P C$ of the inequality (3) there exists a solution $v \in P C$ of problem (1) with

$$
\|u(t, x)-v(t, x)\|_{E} \leq c_{f, g_{k}}(\epsilon) ; \quad(t, x) \in J
$$

Definition $2.7([9,29])$ Problem $(1)$ is Ulam-Hyers-Rassias stable with respect to $(\Phi, \Psi)$ if there exists a real number $c_{f, g_{k}, \Phi}>0$ such that for each $\epsilon>0$ and for each solution $u \in P C$ 
of the inequality (5) there exists a solution $v \in P C$ of problem (1) with

$$
\|u(t, x)-v(t, x)\|_{E} \leq \epsilon c_{f, g_{k}, \Phi}(\Psi+\Phi(t, x)) ; \quad(t, x) \in J .
$$

Definition 2.8 ([9, 29]) Problem (1) is generalized Ulam-Hyers-Rassias stable with respect to $(\Phi, \Psi)$ if there exists a real number $c_{f, g_{k}, \Phi}>0$ such that for each solution $u \in P C$ of the inequality (4) there exists a solution $v \in P C$ of problem (1) with $\|u(t, x)-v(t, x)\|_{E} \leq$ $c_{f, g_{k}, \Phi}(\Psi+\Phi(t, x)) ;(t, x) \in J$.

Remark 2.9 It is clear that: (i) Definition $2.5 \Rightarrow$ Definition 2.6, (ii) Definition $2.7 \Rightarrow$ Definition 2.8, (iii) Definition 2.7 for $\Phi(\cdot, \cdot)=\Psi=1 \Rightarrow$ Definition 2.5 .

Remark 2.10 A function $u \in P C$ is a solution of the inequality (3) if and only if there exist a function $G \in P C$ and a sequence $G_{k} ; k=1, \ldots, m$ in $E$ (which depend on $u$ ) such that

(i) $\|G(t, x)\|_{E} \leq \epsilon$ and $\left\|G_{k}\right\|_{E} \leq \epsilon ; k=1, \ldots, m$,

(ii) ${ }^{c} D_{\theta_{k}}^{r} u(t, x)=f(t, x, u(t, x))+G(t, x)$; if $(t, x) \in I_{k}, k=0, \ldots, m$,

(iii) $u(t, x)=g_{k}(t, x, u(t, x))+G_{k}$; if $(t, x) \in J_{k}, k=1, \ldots, m$,

One can have similar remarks for the inequalities (4) and (5). So, the Ulam stabilities of the impulsive fractional differential equations are some special types of data dependence of the solutions of impulsive fractional differential equations.

In the sequel we will make use of the following generalization of Gronwall's lemma for two independent variables and singular kernel.

Lemma 2.11 (Gronwall lemma [31]) Let $v: J \rightarrow[0, \infty)$ be a real function and $\omega(\cdot, \cdot)$ be a nonnegative, locally integrable function on J. If there are constants $c>0$ and $0<r_{1}, r_{2}<1$ such that

$$
v(t, x) \leq \omega(t, x)+c \int_{0}^{t} \int_{0}^{x} \frac{v(\tau, \xi)}{(t-\tau)^{r_{1}}(x-\xi)^{r_{2}}} d \xi d \tau
$$

then there exists a constant $\delta=\delta\left(r_{1}, r_{2}\right)$ such that

$$
v(t, x) \leq \omega(t, x)+\delta c \int_{0}^{t} \int_{0}^{x} \frac{\omega(\tau, \xi)}{(t-\tau)^{r_{1}}(x-\xi)^{r_{2}}} d \xi d \tau
$$

for every $(t, x) \in J$.

\section{Uniqueness and Ulam stabilities results for finite delay}

In this section, we present conditions for the uniqueness and Ulam stability of problem (1). Consider the Banach space

$$
\begin{aligned}
P C= & \left\{u:[-\alpha, a] \times[-\beta, b] \rightarrow E: u \in C\left(\left(t_{k}, t_{k+1}\right] \times[0, b]\right) ; k=0,1, \ldots, m,\right. \\
& \text { and there exist } u\left(t_{k}^{-}, x\right) \text { and } u\left(t_{k}^{+}, x\right) ; k=1, \ldots, m, \\
& \text { with } \left.u\left(t_{k}^{-}, x\right)=u\left(t_{k}, x\right) \text { for each } x \in[0, b]\right\},
\end{aligned}
$$


with the norm

$$
\|u\|_{P C}=\sup _{(t, x) \in[-\alpha, a] \times[-\beta, b]}\|u(t, x)\|_{E}
$$

By Lemma 2.14 in [1], we conclude to the following lemma.

Lemma 3.1 Let $r_{1}, r_{2} \in(0,1], \mu(t, x)=\varphi(t)+\psi(x)-\varphi(0)$. A function $u \in P C$ is called a solution of the problem (1), if u satisfies

$$
\left\{\begin{aligned}
u(t, x)= & \mu(t, x) \\
& +\int_{0}^{t} \int_{0}^{x} \frac{(t-\tau)^{r_{1}-1}(x-\xi)^{r_{2}-1}}{\Gamma\left(r_{1}\right) \Gamma\left(r_{2}\right)} f\left(\tau, \xi, u_{(\tau, \xi)}\right) d \xi d \tau ; \quad \text { if }(t, x) \in I_{0}, \\
u(t, x)= & \varphi(t)+g_{k}\left(s_{k}, x, u\left(s_{k}, x\right)\right)-g_{k}\left(s_{k}, 0, u\left(s_{k}, 0\right)\right) \\
& +\int_{s_{k}}^{t} \int_{0}^{x} \frac{(t-\tau)^{r_{1}-1}(x-\xi){ }^{r_{2}-1}}{\Gamma\left(r_{1}\right) \Gamma\left(r_{2}\right)} f\left(\tau, \xi, u_{(\tau, \xi)}\right) d \xi d \tau ; \quad \text { if }(t, x) \in I_{k}, k=1, \ldots, m, \\
u(t, x)= & g_{k}(t, x, u(t, x)) ; \quad \text { if }(t, x) \in J_{k}, k=1, \ldots, m, \\
u(t, x)= & \phi(t, x) ; \quad \text { if }(t, x) \in \tilde{J}, \\
u(t, 0)= & \varphi(t) ; \quad t \in[0, a], \quad u(0, x)=\psi(x) ; \quad x \in[0, b] \text { and } \varphi(0)=\psi(0) .
\end{aligned}\right.
$$

Lemma 3.2 If $u \in P C$ is a solution of the inequality (3) then $u$ is a solution of the following integral inequality:

$$
\left\{\begin{array}{l}
\left\|u(t, x)-\mu(t, x)-\int_{0}^{t} \int_{0}^{x} \frac{(t-\tau)^{r_{1}-1}(x-\xi)^{r_{2}-1}}{\Gamma\left(r_{1}\right) \Gamma\left(r_{2}\right)} f\left(\tau, \xi, u_{(\tau, \xi)}\right) d \xi d \tau\right\|_{E} \\
\quad \leq \frac{\epsilon a^{r_{1}} b^{r_{2}}}{\Gamma\left(1+r_{1}\right) \Gamma\left(1+r_{2}\right)} ; \quad \text { if }(t, x) \in I_{0}, \\
\| u(t, x)-\varphi(t)-g_{k}\left(s_{k}, x, u\left(s_{k}, x\right)\right)+g_{k}\left(s_{k}, 0, u\left(s_{k}, 0\right)\right) \\
\quad-\int_{s_{k}}^{t} \int_{0}^{x} \frac{(t-\tau)^{r_{1}-1}(x-\xi)^{r_{2}-1}}{\Gamma\left(r_{1}\right) \Gamma\left(r_{2}\right)} f\left(\tau, \xi, u_{(\tau, \xi)}\right) d \xi d \tau \|_{E} \\
\leq \frac{\left.\epsilon a^{r_{1} b^{r_{2}}}\right)}{\Gamma\left(1+r_{1}\right) \Gamma\left(1+r_{2}\right)} ; \quad \text { if }(t, x) \in I_{k}, k=1, \ldots, m, \\
\left\|u(t, x)-g_{k}(t, x, u(t, x))\right\|_{E} \leq \epsilon ; \quad \text { if }(t, x) \in J_{k}, k=1, \ldots, m .
\end{array}\right.
$$

Proof By Remark 2.10 we have

$$
\left\{\begin{array}{l}
{ }^{c} D_{\theta_{k}}^{r} u(t, x)=f\left(t, x, u_{(t, x)}\right)+G(t, x) ; \quad \text { if }(t, x) \in I_{k}, k=0, \ldots, m, \\
u(t, x)=g_{k}(t, x, u(t, x))+G_{k} ; \quad \text { if }(t, x) \in J_{k}, k=1, \ldots, m .
\end{array}\right.
$$

Then

$$
\left\{\begin{aligned}
u(t, x)= & \mu(t, x) \\
& +\int_{0}^{t} \int_{0}^{x} \frac{(t-\tau)^{r_{1}-1}(x-\xi)^{r_{2}-1}}{\Gamma\left(r_{1}\right) \Gamma\left(r_{2}\right)}\left(f\left(\tau, \xi, u_{(\tau, \xi)}\right)+G(\tau, \xi)\right) d \xi d \tau ; \quad \text { if }(t, x) \in I_{0}, \\
u(t, x)= & \varphi(t)+g_{k}\left(s_{k}, x, u\left(s_{k}, x\right)\right)-g_{k}\left(s_{k}, 0, u\left(s_{k}, 0\right)\right) \\
& +\int_{s_{k}}^{t} \int_{0}^{x} \frac{(t-\tau)^{r_{1}-1}(x-\xi)^{r_{2}-1}}{\Gamma\left(r_{1}\right) \Gamma\left(r_{2}\right)}\left(f\left(\tau, \xi, u_{(\tau, \xi)}\right)+G(\tau, \xi)\right) d \xi d \tau ; \\
& \text { if }(t, x) \in I_{k}, k=1, \ldots, m, \\
u(t, x)= & g_{k}(t, x, u(t, x))+G_{k} ; \quad \text { if }(t, x) \in J_{k}, k=1, \ldots, m .
\end{aligned}\right.
$$


Thus, it follows that

$$
\left\{\begin{array}{l}
\left\|u(t, x)-\mu(t, x)-\int_{0}^{t} \int_{0}^{x} \frac{(t-\tau)^{r_{1}-1}(x-\xi)^{r_{2}-1}}{\Gamma\left(r_{1}\right) \Gamma\left(r_{2}\right)} f\left(\tau, \xi, u_{(\tau, \xi)}\right) d \xi d \tau\right\|_{E} \\
\quad=\left\|\int_{0}^{t} \int_{0}^{x} \frac{(t-\tau)^{r_{1}-1}(x-\xi)^{r_{2}-1}}{\Gamma\left(r_{1}\right) \Gamma\left(r_{2}\right)} G(\tau, \xi) d \xi d \tau\right\|_{E} ; \quad \text { if }(t, x) \in I_{0}, \\
\| u(t, x)-\varphi(t)-g_{k}\left(s_{k}, x, u\left(s_{k}, x\right)\right)+g_{k}\left(s_{k}, 0, u\left(s_{k}, 0\right)\right) \\
\quad-\int_{s_{k}}^{t} \int_{0}^{x} \frac{(t-\tau)^{r_{1}-1}(x-\xi)^{r_{2}-1}}{\Gamma\left(r_{1}\right) \Gamma\left(r_{2}\right)} f\left(\tau, \xi, u_{(\tau, \xi)}\right) d \xi d \tau \|_{E} \\
\quad=\left\|\int_{0}^{t} \int_{0}^{x} \frac{(t-\tau)^{r_{1}-1}(x-\xi)^{r_{2}-1}}{\Gamma\left(r_{1}\right) \Gamma\left(r_{2}\right)} G(\tau, \xi) d \xi d \tau\right\|_{E} ; \quad \text { if }(t, x) \in I_{k}, k=1, \ldots, m, \\
\left\|u(t, x)-g_{k}(t, x, u(t, x))\right\|_{E}=\left\|G_{k}\right\|_{E} ; \quad \text { if }(t, x) \in J_{k}, k=1, \ldots, m .
\end{array}\right.
$$

Hence, we obtain (7).

Remark 3.3 We have similar results for the solutions of the inequalities (4) and (5).

Theorem 3.4 Assume that the following hypotheses hold:

$\left(H_{1}\right)$ There exists a constant $l_{f}>0$ such that

$$
\|f(t, x, u)-f(t, x, \bar{u})\|_{E} \leq l_{f}\|u-\bar{u}\|_{\mathcal{C}}
$$

for each $(t, x) \in J$, and each $u, \bar{u} \in \mathcal{C}$.

$\left(H_{2}\right)$ There exist constants $l_{g_{k}}>0 ; k=1, \ldots, m$, such that

$$
\left\|g_{k}(t, x, u)-g_{k}(t, x, \bar{u})\right\|_{E} \leq l_{g_{k}}\|u-\bar{u}\|_{E}
$$

for each $(t, x) \in J_{k}$, and each $u, \bar{u} \in E, k=1, \ldots, m$.

If

$$
\ell:=2 l_{g}+\frac{l_{f} a^{r_{1}} b^{r_{2}}}{\Gamma\left(1+r_{1}\right) \Gamma\left(1+r_{2}\right)}<1,
$$

where $l_{g}=\max _{k=1, \ldots, m} l_{g_{k}}$, then the problem (1) has a unique solution on J.

Furthermore, if the following hypothesis holds:

$\left(H_{3}\right)$ There exists $\lambda_{\Phi}>0$ such that, for each $(t, x) \in J$, we have

$$
I_{\theta_{k}}^{r} \Phi(t, x) \leq \lambda_{\Phi} \Phi(t, x) ; \quad k=0, \ldots, m
$$

then the problem (1) is generalized Ulam-Hyers-Rassias stable.

Proof Consider the operator $N: P C \rightarrow P C$ defined by

$$
\left\{\begin{aligned}
(N u)(t, x)= & \mu(t, x) \\
& +\int_{0}^{t} \int_{0}^{x} \frac{(t-\tau)^{r_{1}-1}(x-\xi)^{r_{2}-1}}{\Gamma\left(r_{1}\right) \Gamma\left(r_{2}\right)} f\left(\tau, \xi, u_{(\tau, \xi)}\right) d \xi d \tau ; \quad \text { if }(t, x) \in I_{0}, \\
(N u)(t, x)= & \varphi(t)+g_{k}\left(s_{k}, x, u\left(s_{k}, x\right)\right)-g_{k}\left(s_{k}, 0, u\left(s_{k}, 0\right)\right) \\
& +\int_{s_{k}}^{t} \int_{0}^{x} \frac{(t-\tau)^{r_{1}-1}(x-\xi)^{r_{2}-1}}{\Gamma\left(r_{1}\right) \Gamma\left(r_{2}\right)} f\left(\tau, \xi, u_{(\tau, \xi)}\right) d \xi d \tau ; \quad \text { if }(t, x) \in I_{k}, k=1, \ldots, m, \\
(N u)(t, x)= & g_{k}(t, x, u(t, x)) ; \quad \text { if }(t, x) \in J_{k}, k=1, \ldots, m, \\
(N u)(t, x)= & \phi(t, x) ; \quad \text { if }(t, x) \in \tilde{J} .
\end{aligned}\right.
$$


Clearly, the fixed points of the operator $N$ are solution of the problem (1). We shall use the Banach contraction principle to prove that $N$ has a fixed point. $N$ is a contraction. Let $u, v \in P C$, then, for each $(t, x) \in J$, we have

$$
\left\{\begin{array}{l}
\|(N u)(t, x)-(N v)(t, x)\|_{E} \\
\leq\left\|\int_{0}^{t} \int_{0}^{x} \frac{(t-\tau)^{r_{1}-1}(x-\xi)^{r_{2}-1}}{\Gamma\left(r_{1}\right) \Gamma\left(r_{2}\right)}\left[f\left(\tau, \xi, u_{(\tau, \xi)}\right)-f\left(\tau, \xi, v_{(\tau, \xi)}\right)\right] d \xi d \tau\right\|_{E} ; \\
\quad \text { if }(t, x) \in I_{0}, \\
\|(N u)(t, x)-(N v)(t, x)\|_{E} \\
\leq\left\|g_{k}\left(s_{k}, x, u\left(s_{k}, x\right)\right)-g_{k}\left(s_{k}, x, v\left(s_{k}, x\right)\right)\right\|_{E} \\
\quad+\left\|g_{k}\left(s_{k}, 0, u\left(s_{k}, 0\right)\right)-g_{k}\left(s_{k}, 0, v\left(s_{k}, 0\right)\right)\right\|_{E} \\
\quad+\left\|\int_{s_{k}}^{t} \int_{0}^{x} \frac{(t-\tau)^{r_{1}-1}(x-\xi)^{r_{2}-1}}{\Gamma\left(r_{1}\right) \Gamma\left(r_{2}\right)}\left[f\left(\tau, \xi, u_{(\tau, \xi)}\right)-f\left(\tau, \xi, v_{(\tau, \xi)}\right)\right] d \xi d \tau\right\|_{E} ; \\
\quad \text { if }(t, x) \in I_{k}, k=1, \ldots, m, \\
\|(N u)(t, x)-(N v)(t, x)\|_{E} \\
=\left\|g_{k}(t, x, u(t, x))-g_{k}(t, x, v(t, x))\right\|_{E} ; \quad \text { if }(t, x) \in J_{k}, k=1, \ldots, m .
\end{array}\right.
$$

Thus, we get

$$
\left\{\begin{array}{l}
\|(N u)(t, x)-(N v)(t, x)\|_{E} \\
\quad \leq \int_{0}^{t} \int_{0}^{x} \frac{(t-\tau) r^{r_{1}-1}(x-\xi)^{r_{2}-1}}{\Gamma\left(r_{1}\right) \Gamma\left(r_{2}\right)} l_{f}\|u-v\|_{\mathcal{C}} d \xi d \tau \\
\quad \leq \frac{l_{f} a^{r_{1}} b^{r_{2}}}{\Gamma\left(1+r_{1}\right) \Gamma\left(1+r_{2}\right)}\|u-v\|_{P C} ; \quad \text { if }(t, x) \in I_{0}, \\
\|(N u)(t, x)-(N v)(t, x)\|_{E} \\
\quad \leq 2 l_{g}\|u-v\|_{P C}+\int_{s_{k}}^{t} \int_{0}^{x} \frac{(t-\tau)^{r_{1}-1}(x-\xi)^{r_{2}-1}}{\Gamma\left(r_{1}\right) \Gamma\left(r_{2}\right)} l_{f}\|u-v\|_{\mathcal{C}} d \xi d \tau \\
\quad \leq\left(2 l_{g}+\frac{l_{f} a^{r_{1}} b^{r_{2}}}{\Gamma\left(1+r_{1}\right) \Gamma\left(1+r_{2}\right)}\right)\|u-v\|_{P C} ; \quad \text { if }(t, x) \in I_{k}, k=1, \ldots, m, \\
\|(N u)(t, x)-(N v)(t, x)\|_{E} \leq l_{g}\|u-v\|_{P C} ; \quad \text { if }(t, x) \in J_{k}, k=1, \ldots, m .
\end{array}\right.
$$

Hence

$$
\|N(u)-N(v)\|_{P C} \leq \ell\|u-v\|_{P C}
$$

By the condition (8), we conclude that $N$ is a contraction. As a consequence of Banach fixed point theorem, we deduce that $N$ has a unique fixed point $v$ which is a solution of the problem (1). Then we have

$$
\left\{\begin{aligned}
v(t, x)= & \mu(t, x) \\
& +\int_{0}^{t} \int_{0}^{x} \frac{(t-\tau)^{r_{1}-1}(x-\xi)^{r_{2}-1}}{\Gamma\left(r_{1}\right) \Gamma\left(r_{2}\right)} f\left(\tau, \xi, v_{(\tau, \xi)}\right) d \xi d \tau ; \quad \text { if }(t, x) \in I_{0}, \\
v(t, x)= & \varphi(t)+g_{k}\left(s_{k}, x, v\left(s_{k}, x\right)\right)-g_{k}\left(s_{k}, 0, v\left(s_{k}, 0\right)\right) \\
& +\int_{s_{k}}^{t} \int_{0}^{x} \frac{(t-\tau)^{r_{1}-1}(x-\xi)^{r_{2}-1}}{\Gamma\left(r_{1}\right) \Gamma\left(r_{2}\right)} f\left(\tau, \xi, v_{(\tau, \xi)}\right) d \xi d \tau ; \quad \text { if }(t, x) \in I_{k}, k=1, \ldots, m, \\
v(t, x)= & g_{k}(t, x, v(t, x)) ; \quad \text { if }(t, x) \in J_{k}, k=1, \ldots, m .
\end{aligned}\right.
$$


Let $u \in P C$ be a solution of the inequality (4). By the differential of this inequality, for each $(t, x) \in J$, we have

$$
\left\{\begin{array}{l}
\left\|u(t, x)-\mu(t, x)-\int_{0}^{t} \int_{0}^{x} \frac{(t-\tau)^{r_{1}-1}(x-\xi)^{r_{2}-1}}{\Gamma\left(r_{1}\right) \Gamma\left(r_{2}\right)} f\left(\tau, \xi, u_{(\tau, \xi)}\right) d \xi d \tau\right\|_{E} \\
\quad \leq\left\|\int_{0}^{t} \int_{0}^{x} \frac{(t-\tau)^{r_{1}-1}(x-\xi)^{r_{2}-1}}{\Gamma\left(r_{1}\right) \Gamma\left(r_{2}\right)} \Phi(\tau, \xi) d \xi d \tau\right\|_{E} ; \quad \text { if }(t, x) \in I_{0}, \\
\| u(t, x)-\varphi(t)-g_{k}\left(s_{k}, x, u\left(s_{k}, x\right)\right)+g_{k}\left(s_{k}, 0, u\left(s_{k}, 0\right)\right) \\
\quad-\int_{s_{k}}^{t} \int_{0}^{x} \frac{(t-\tau)^{r_{1}-1}(x-\xi)^{r_{2}-1}}{\Gamma\left(r_{1}\right) \Gamma\left(r_{2}\right)} f\left(\tau, \xi, u_{(\tau, \xi)}\right) d \xi d \tau \|_{E} \\
\quad \leq\left\|\int_{0}^{t} \int_{0}^{x} \frac{(t-\tau)^{r_{1}-1}(x-\xi)^{2}{ }^{2}-1}{\Gamma\left(r_{1}\right) \Gamma\left(r_{2}\right)} \Phi(\tau, \xi) d \xi d \tau\right\|_{E} ; \quad \text { if }(t, x) \in I_{k}, k=1, \ldots, m, \\
\left\|u(t, x)-g_{k}(t, x, u(t, x))\right\|_{E} \leq \Psi ; \quad \text { if }(t, x) \in J_{k}, k=1, \ldots, m .
\end{array}\right.
$$

Thus, by $\left(H_{3}\right)$ for each $(t, x) \in J$, we get

$$
\left\{\begin{array}{l}
\left\|u(t, x)-\mu(t, x)-\int_{0}^{t} \int_{0}^{x} \frac{(t-\tau)^{r_{1}-1}(x-\xi)^{r_{2}-1}}{\Gamma\left(r_{1}\right) \Gamma\left(r_{2}\right)} f\left(\tau, \xi, u_{(\tau, \xi)}\right) d \xi d \tau\right\|_{E} \\
\quad \leq \lambda_{\Phi} \Phi(t, x) ; \quad \text { if }(t, x) \in I_{0}, \\
\| u(t, x)-\varphi(t)-g_{k}\left(s_{k}, x, u\left(s_{k}, x\right)\right)+g_{k}\left(s_{k}, 0, u\left(s_{k}, 0\right)\right) \\
\quad-\int_{s_{k}}^{t} \int_{0}^{x} \frac{(t-\tau)^{r_{1}-1}(x-\xi)^{r_{2}-1}}{\Gamma\left(r_{1}\right) \Gamma\left(r_{2}\right)} f\left(\tau, \xi, u_{(\tau, \xi)}\right) d \xi d \tau \|_{E} \\
\leq \lambda_{\Phi} \Phi(t, x) ; \quad \text { if }(t, x) \in I_{k}, k=1, \ldots, m, \\
\left\|u(t, x)-g_{k}(t, x, u(t, x))\right\|_{E} \leq \Psi ; \quad \text { if }(t, x) \in J_{k}, k=1, \ldots, m .
\end{array}\right.
$$

Hence

$$
\left\{\begin{array}{l}
\|u(t, x)-v(t, x)\|_{E} \\
\leq \lambda_{\Phi} \Phi(t, x) \\
\quad+\int_{0}^{t} \int_{0}^{x} \frac{(t-\tau)^{r_{1}-1}(x-\xi)^{r_{2}-1}}{\Gamma\left(r_{1}\right) \Gamma\left(r_{2}\right)}\left\|f\left(\tau, \xi, u_{(\tau, \xi)}\right)-f\left(\tau, \xi, v_{(\tau, \xi)}\right)\right\|_{E} d \xi d \tau ; \\
\quad \text { if }(t, x) \in I_{0}, \\
\|u(t, x)-v(t, x)\|_{E} \\
\leq \lambda_{\Phi} \Phi(t, x)+2 l_{g}\|u(t, x)-v(t, x)\|_{E} \\
\quad+\int_{s_{k}}^{t} \int_{0}^{x} \frac{(t-\tau)^{r_{1}-1}(x-\xi)^{r_{2}-1}}{\Gamma\left(r_{1}\right) \Gamma\left(r_{2}\right)}\left\|f\left(\tau, \xi, u_{(\tau, \xi)}\right)-f\left(\tau, \xi, v_{(\tau, \xi)}\right)\right\|_{E} d \xi d \tau ; \\
\quad \text { if }(t, x) \in I_{k}, k=1, \ldots, m, \\
\|u(t, x)-v(t, x)\|_{E} \\
\leq \Psi+\left\|g_{k}(t, x, u(t, x))-g_{k}(t, x, v(t, x))\right\|_{E} \\
\leq \\
\quad \Psi+l_{g}\|u(t, x)-v(t, x)\|_{E} ; \quad \text { if }(t, x) \in J_{k}, k=1, \ldots, m .
\end{array}\right.
$$

For each $(t, x) \in I_{0}$, we have

$$
\|u(t, x)-v(t, x)\|_{E} \leq \lambda_{\Phi} \Phi(t, x)+l_{f} \int_{0}^{t} \int_{0}^{x} \frac{(t-\tau)^{r_{1}-1}(x-\xi)^{r_{2}-1}}{\Gamma\left(r_{1}\right) \Gamma\left(r_{2}\right)}\left\|u_{(\tau, \xi)}-v_{(\tau, \xi)}\right\|_{\mathcal{C}} d \xi d \tau .
$$

We consider the function $\gamma$ defined by

$$
\gamma(t, x)=\sup \{\|u(\tau, \xi)-v(\tau, \xi)\|:-\alpha \leq \tau \leq t,-\beta \leq \xi \leq x\} ; \quad(t, x) \in J .
$$


Let $\left(t^{*}, x^{*}\right) \in[-\alpha, x] \times[-\beta, y]$ be such that $\gamma(t, x)=\left\|u\left(t^{*}, x^{*}\right)-v\left(t^{*}, x^{*}\right)\right\|_{E}$. If $\left(t^{*}, x^{*}\right) \in \tilde{J}$, then $\gamma(t, x)=0$. Now, if $\left(t^{*}, x^{*}\right) \in J$, then by the previous inequality, we have, for $(t, x) \in J$,

$$
\gamma(t, x) \leq \lambda_{\Phi} \Phi(t, x)+l_{f} \int_{0}^{t} \int_{0}^{x} \frac{(t-\tau)^{r_{1}-1}(x-\xi)^{r_{2}-1}}{\Gamma\left(r_{1}\right) \Gamma\left(r_{2}\right)} \gamma(t, x) d \xi d \tau
$$

From Lemma 2.11, there exists a constant $\delta_{1}:=\delta_{1}\left(r_{1}, r_{2}\right)$ such that

$$
\begin{aligned}
\gamma(t, x) & \leq \lambda_{\Phi}\left(\Phi(t, x)+l_{f} \delta_{1} I_{\theta}^{r} \Phi(t, x)\right) \\
& \leq \lambda_{\Phi}\left(1+l_{f} \delta_{1} \lambda_{\Phi}\right) \Phi(t, x) \\
& :=c_{1, f, g_{k}, \Phi} \Phi(t, x) .
\end{aligned}
$$

Since for every $(t, x) \in I_{0},\left\|u_{(t, x)}\right\|_{\mathcal{C}} \leq \gamma(t, x)$, we get

$$
\|u(t, x)-v(t, x)\|_{E} \leq c_{1, f, g_{k}, \Phi}(\Psi+\Phi(t, x))
$$

Now, for each $(t, x) \in I_{k}, k=1, \ldots, m$, we have

$$
\begin{aligned}
& \|u(t, x)-v(t, x)\|_{E} \\
& \leq \lambda_{\Phi} \Phi(t, x)+2 l_{g}\|u(t, x)-v(t, x)\|_{E} \\
& \quad+l_{f} \int_{s_{k}}^{t} \int_{0}^{x} \frac{(t-\tau)^{r_{1}-1}(x-\xi)^{r_{2}-1}}{\Gamma\left(r_{1}\right) \Gamma\left(r_{2}\right)}\|u(\tau, \xi)-v(\tau, \xi)\|_{E} d \xi d \tau .
\end{aligned}
$$

Then we obtain

$$
\begin{aligned}
& \|u(t, x)-v(t, x)\|_{E} \\
& \quad \leq \frac{\lambda_{\Phi}}{1-2 l_{g}} \Phi(t, x)+\frac{l_{f}}{1-2 l_{g}} \int_{s_{k}}^{t} \int_{0}^{x} \frac{(t-\tau)^{r_{1}-1}(x-\xi)^{r_{2}-1}}{\Gamma\left(r_{1}\right) \Gamma\left(r_{2}\right)}\|u(\tau, \xi)-v(\tau, \xi)\|_{E} d \xi d \tau .
\end{aligned}
$$

Again, from Lemma 2.11, there exists a constant $\delta_{2}:=\delta_{2}\left(r_{1}, r_{2}\right)$ such that

$$
\begin{aligned}
\|u(t, x)-v(t, x)\|_{E} & \leq \frac{\lambda_{\Phi}}{1-2 l_{g}}\left(\Phi(t, x)+\frac{l_{f} \delta_{2}}{1-2 l_{g}} I_{\theta_{k}}^{r} \Phi(t, x)\right) \\
& \leq \frac{\lambda_{\Phi}}{1-2 l_{g}}\left(1+\frac{l_{f} \delta_{2} \lambda_{\Phi}}{1-2 l_{g}}\right) \Phi(t, x) \\
& :=c_{2, f, g_{k}, \Phi} \Phi(t, x) .
\end{aligned}
$$

Hence, for each $(t, x) \in I_{k}, k=1, \ldots, m$, we get

$$
\|u(t, x)-v(t, x)\|_{E} \leq c_{2, f, g_{k}, \Phi}(\Psi+\Phi(t, x)) .
$$

Now, for each $(t, x) \in J_{k}, k=1, \ldots, m$, we have

$$
\|u(t, x)-v(t, x)\|_{E} \leq \Psi+l_{g}\|u(t, x)-v(t, x)\|_{E} .
$$


This gives

$$
\|u(t, x)-v(t, x)\|_{E} \leq \frac{\Psi}{1-l_{g}}:=c_{3, f, g_{k}, \Phi} \Psi .
$$

Thus, for each $(t, x) \in J_{k}, k=1, \ldots, m$, we get

$$
\|u(t, x)-v(t, x)\|_{E} \leq c_{3, f, g_{k}, \Phi}(\Psi+\Phi(t, x)) .
$$

Set $c_{f, g_{k}, \Phi}:=\max _{i \in\{1,2,3\}} c_{i, f, g_{k}, \Phi}$. Hence, for each $(t, x) \in J$, we obtain

$$
\|u(t, x)-v(t, x)\|_{E} \leq c_{f, g_{k}, \Phi}(\Psi+\Phi(t, x)) .
$$

Consequently, problem (1) is generalized Ulam-Hyers-Rassias stable.

\section{The phase space $\mathcal{B}$}

The notation of the phase space $\mathcal{B}$ plays an important role in the study of both qualitative and quantitative theory for functional differential equations. A usual choice is a seminormed space satisfying suitable axioms, which was introduced by Hale and Kato [32]. For any $(t, x) \in J$ denote $\mathcal{E}_{(t, x)}:=[0, t] \times\{0\} \cup\{0\} \times[0, x]$, furthermore in the case $t=a$, $x=b$ we write simply $\mathcal{E}$. Consider the space $\left(\mathcal{B},\|(\cdot, \cdot)\|_{\mathcal{B}}\right)$ is a seminormed linear space of functions mapping $(-\infty, 0] \times(-\infty, 0]$ into $E$, and satisfying the following fundamental axioms which were adapted from those introduced by Hale and Kato for ordinary differential functional equations:

$\left(A_{1}\right)$ If $z:(-\infty, a] \times(-\infty, b] \rightarrow E$ continuous on $J$ and $z_{(t, x)} \in \mathcal{B}$, for all $(t, x) \in \mathcal{E}$, then there are constants $H, K, M>0$ such that for any $(t, x) \in J$ the following conditions hold:

(i) $z_{(t, x)}$ is in $\mathcal{B}$,

(ii) $\|z(t, x)\| \leq H\left\|z_{(t, x)}\right\|_{\mathcal{B}}$,

(iii) $\left\|z_{(t, x)}\right\|_{\mathcal{B}} \leq K \sup _{(\tau, \xi) \in[0, t] \times[0, x]}\|z(\tau, \xi)\|+M \sup _{(\tau, \xi) \in E_{(t, x)}}\left\|z_{(\tau, \xi)}\right\|_{\mathcal{B}}$,

$\left(A_{2}\right)$ for the function $z(\cdot, \cdot)$ in $\left(A_{1}\right), z_{(t, x)}$ is a $\mathcal{B}$-valued continuous function on $J$,

$\left(A_{3}\right)$ the space $\mathcal{B}$ is complete.

Now, we present some examples of phase spaces [33, 34].

Example 4.1 Let $\mathcal{B}$ be the set of all functions $\phi:(-\infty, 0] \times(-\infty, 0] \rightarrow E$ which are continuous on $[-\alpha, 0] \times[-\beta, 0], \alpha, \beta \geq 0$, with the seminorm

$$
\|\phi\|_{\mathcal{B}}=\sup _{(s, t) \in[-\alpha, 0] \times[-\beta, 0]}\|\phi(s, t)\| .
$$

Then we have $H=K=M=1$. The quotient space $\widehat{\mathcal{B}}=\mathcal{B} /\|\cdot\|_{\mathcal{B}}$ is isometric to the space $\mathcal{C}$, this means that partial differential functional equations with finite delay are included in our axiomatic model.

Example 4.2 Let $\gamma$ be a real constant and Let $C_{\gamma}$ be the set of all continuous functions

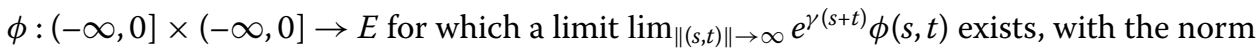

$$
\|\phi\|_{C_{\gamma}}=\sup _{(s, t) \in(-\infty, 0] \times(-\infty, 0]} e^{\gamma(s+t)}\|\phi(s, t)\| .
$$

Then we have $H=1$ and $K=M=\max \left\{e^{-\gamma(a+b)}, 1\right\}$. 
Example 4.3 Let $\alpha, \beta, \gamma \geq 0$ and let

$$
\|\phi\|_{C L_{\gamma}}=\sup _{(s, t) \in[-\alpha, 0] \times[-\beta, 0]}\|\phi(s, t)\|+\int_{-\infty}^{0} \int_{-\infty}^{0} e^{\gamma(s+t)}\|\phi(s, t)\| d t d s
$$

be the seminorm for the space $C L_{\gamma}$ of all functions $\phi:(-\infty, 0] \times(-\infty, 0] \rightarrow E$ which are continuous on $[-\alpha, 0] \times[-\beta, 0]$ measurable on $(-\infty,-\alpha] \times(-\infty, 0] \cup(-\infty, 0] \times(-\infty,-\beta]$, and such that $\|\phi\|_{C L_{\gamma}}<\infty$. Then

$$
H=1, \quad K=\int_{-\alpha}^{0} \int_{-\beta}^{0} e^{\gamma(s+t)} d t d s, \quad M=2 .
$$

\section{Uniqueness and Ulam stabilities results for infinite delay}

In this section, we present conditions for the Ulam stability of problem (2). Consider the space

$$
\Omega:=\left\{u:(-\infty, a] \times(-\infty, b] \rightarrow E: u_{(t, x)} \in \mathcal{B} \text { for }(t, x) \in \mathcal{E} \text { and }\left.u\right|_{J} \in P C\right\}
$$

Theorem 5.1 Assume that the following hypotheses hold:

$\left(H_{1}^{\prime}\right)$ There exists a constant $l_{f}^{\prime}>0$ such that

$$
\|f(t, x, u)-f(t, x, \bar{u})\|_{E} \leq l_{f}\|u-\bar{u}\|_{\mathcal{B}},
$$

for each $(t, x) \in J$, and each $u, \bar{u} \in \mathcal{B}$.

$\left(H_{2}^{\prime}\right)$ There exist constants $l_{g_{k}}^{\prime}>0 ; k=1, \ldots, m$, such that

$$
\left\|g_{k}(t, x, u)-g_{k}(t, x, \bar{u})\right\|_{E} \leq l_{g_{k}}^{\prime}\|u-\bar{u}\|_{E}
$$

for each $(t, x) \in J_{k}$, and each $u, \bar{u} \in E, k=1, \ldots, m$.

If

$$
\ell^{\prime}:=2 l_{g}^{\prime}+\frac{K l_{f}^{\prime} a^{r_{1}} b^{r_{2}}}{\Gamma\left(1+r_{1}\right) \Gamma\left(1+r_{2}\right)}<1
$$

where $l_{g}^{\prime}=\max _{k=1, \ldots, m} l_{g_{k}}^{\prime}$, then the problem (2) has a unique solution on $(-\infty, a] \times(-\infty, b]$.

Furthermore, if the hypothesis $\left(H_{3}\right)$ holds, then the problem (2) is generalized UlamHyers-Rassias stable.

Proof Consider the operator $N^{\prime}: \Omega \rightarrow \Omega$ defined by

$$
\left\{\begin{aligned}
\left(N^{\prime} u\right)(t, x)= & \mu(t, x) \\
& +\int_{0}^{t} \int_{0}^{x} \frac{(t-\tau)^{r_{1}-1}(x-\xi)^{r_{2}-1}}{\Gamma\left(r_{1}\right) \Gamma\left(r_{2}\right)} f\left(\tau, \xi, u_{(\tau, \xi)}\right) d \xi d \tau ; \quad \text { if }(t, x) \in I_{0}, \\
\left(N^{\prime} u\right)(t, x)= & \varphi(t)+g_{k}\left(s_{k}, x, u\left(s_{k}, x\right)\right)-g_{k}\left(s_{k}, 0, u\left(s_{k}, 0\right)\right) \\
& +\int_{s_{k}}^{t} \int_{0}^{x} \frac{(t-\tau)^{r_{1}-1}(x-\xi)^{r_{2}-1}}{\Gamma\left(r_{1}\right) \Gamma\left(r_{2}\right)} f\left(\tau, \xi, u_{(\tau, \xi)}\right) d \xi d \tau ; \quad \text { if }(t, x) \in I_{k}, k=1, \ldots, m, \\
\left(N^{\prime} u\right)(t, x)= & g_{k}(t, x, u(t, x)) ; \quad \text { if }(t, x) \in J_{k}, k=1, \ldots, m, \\
\left(N^{\prime} u\right)(t, x)= & \phi(t, x) ; \quad \text { if }(t, x) \in \tilde{J}^{\prime} .
\end{aligned}\right.
$$


Let $v(\cdot, \cdot):(-\infty, a] \times(-\infty, b] \rightarrow E$ be a function defined by

$$
\begin{cases}v(t, x)=\mu(t, x) ; & \text { if }(t, x) \in J, \\ v(t, x)=\phi(t, x) ; & \text { if }(t, x) \in \tilde{J}^{\prime} .\end{cases}
$$

Then $v_{(t, x)}=\phi$ for all $(t, x) \in \mathcal{E}$. For each $w \in C(J)$ with $w(t, x)=0 ;(t, x) \in \mathcal{E}$ we denote by $\bar{w}$ the function defined by

$$
\left\{\begin{array}{l}
\bar{w}(t, x)=w(t, x) ; \quad \text { if }(t, x) \in J \\
\bar{w}(t, x)=0 ; \quad \text { if }(t, x) \in \tilde{J}^{\prime} .
\end{array}\right.
$$

If $u(\cdot, \cdot)$ satisfies

$$
\left\{\begin{aligned}
u(t, x)= & \mu(t, x) \\
& +\int_{0}^{t} \int_{0}^{x} \frac{(t-\tau)^{r_{1}-1}(x-\xi)^{r_{2}-1}}{\Gamma\left(r_{1}\right) \Gamma\left(r_{2}\right)} f\left(\tau, \xi, u_{(\tau, \xi)}\right) d \xi d \tau ; \quad \text { if }(t, x) \in I_{0}, \\
u(t, x)= & \varphi(t)+g_{k}\left(s_{k}, x, u\left(s_{k}, x\right)\right)-g_{k}\left(s_{k}, 0, u\left(s_{k}, 0\right)\right) \\
& +\int_{s_{k}}^{t} \int_{0}^{x} \frac{(t-\tau)^{r_{1}-1}(x-\xi)^{r_{2}-1}}{\Gamma\left(r_{1}\right) \Gamma\left(r_{2}\right)} f\left(\tau, \xi, u_{(\tau, \xi)}\right) d \xi d \tau ; \quad \text { if }(t, x) \in I_{k}, k=1, \ldots, m, \\
u(t, x)= & g_{k}(t, x, u(t, x)) ; \quad \text { if }(t, x) \in J_{k}, k=1, \ldots, m,
\end{aligned}\right.
$$

then we can decompose $u(\cdot, \cdot)$ as $u(t, x)=\bar{w}(t, x)+v(t, x) ;(t, x) \in J$, which implies $u_{(t, x)}=$ $\bar{w}_{(t, x)}+v_{(t, x)}$, for every $(t, x) \in J$, and the function $w(\cdot, \cdot)$ satisfies

$$
\left\{\begin{aligned}
w(t, x)= & \int_{0}^{t} \int_{0}^{x} \frac{(t-\tau)^{r_{1}-1}(x-\xi) r^{r_{2}-1}}{\Gamma\left(r_{1}\right) \Gamma\left(r_{2}\right)} f\left(\tau, \xi, \bar{w}_{(\tau, \xi)}+v_{(\tau, \xi)}\right) d \xi d \tau ; \quad \text { if }(t, x) \in I_{0}, \\
w(t, x)= & \varphi(t)+g_{k}\left(s_{k}, x, \bar{w}\left(s_{k}, x\right)+v\left(s_{k}, x\right)\right)-g_{k}\left(s_{k}, 0, \bar{w}\left(s_{k}, 0\right)+v\left(s_{k}, 0\right)\right) \\
& +\int_{s_{k}}^{t} \int_{0}^{x} \frac{(t-\tau)^{r_{1}-1}(x-\xi) r_{2}-1}{\Gamma\left(r_{1}\right) \Gamma\left(r_{2}\right)} f\left(\tau, \xi, \bar{w}_{(\tau, \xi)}+v_{(\tau, \xi)}\right) d \xi d \tau ; \quad \text { if }(t, x) \in I_{k}, k=1, \ldots, m, \\
w(t, x)= & g_{k}(t, x, \bar{w}(t, x)+v(t, x)) ; \quad \text { if }(t, x) \in J_{k}, k=1, \ldots, m .
\end{aligned}\right.
$$

Set

$$
C_{0}=\{w \in P C: w(t, x)=0 \text { for }(t, x) \in \mathcal{E}\},
$$

and let $\|\cdot\|_{(a, b)}$ be the seminorm in $C_{0}$ defined by

$$
\|w\|_{(a, b)}=\sup _{(t, x) \in E}\left\|w_{(t, x)}\right\|_{\mathcal{B}}+\sup _{(t, x) \in J}\|w(t, x)\|=\sup _{(t, x) \in J}\|w(t, x)\| ; \quad w \in C_{0} .
$$

$C_{0}$ is a Banach space with norm $\|\cdot\|_{(a, b)}$. Let the operator $P: C_{0} \rightarrow C_{0}$ be defined by

$$
\left\{\begin{aligned}
(P w)(t, x)= & \int_{0}^{t} \int_{0}^{x} \frac{(t-\tau)^{r_{1}-1}(x-\xi)^{r_{2}-1}}{\Gamma\left(r_{1}\right) \Gamma\left(r_{2}\right)} f\left(\tau, \xi, \bar{w}_{(\tau, \xi)}+v_{(\tau, \xi)}\right) d \xi d \tau ; \quad \text { if }(t, x) \in I_{0} \\
(P w)(t, x)= & \varphi(t)+g_{k}\left(s_{k}, x, \bar{w}\left(s_{k}, x\right)+v\left(s_{k}, x\right)\right)-g_{k}\left(s_{k}, 0, \bar{w}\left(s_{k}, 0\right)+v\left(s_{k}, 0\right)\right) \\
& +\int_{s_{k}}^{t} \int_{0}^{x} \frac{(t-\tau)^{r_{1}-1}(x-\xi)^{r_{2}-1}}{\Gamma\left(r_{1}\right) \Gamma\left(r_{2}\right)} f\left(\tau, \xi, \bar{w}_{(\tau, \xi)}+v_{(\tau, \xi)}\right) d \xi d \tau ; \\
& \text { if }(t, x) \in I_{k}, k=1, \ldots, m, \\
(P w)(t, x)= & g_{k}(t, x, \bar{w}(t, x)+v(t, x)) ; \quad \text { if }(t, x) \in J_{k}, k=1, \ldots, m .
\end{aligned}\right.
$$


Then the operator $N^{\prime}$ has a fixed point is equivalent to $P$ has a fixed point. We shall show that $P: C_{0} \rightarrow C_{0}$ is a contraction map. Indeed, consider $w, w^{*} \in C_{0}$. Then, for each $(t, x) \in$ $J$, we have

$$
\left\{\begin{array}{l}
\left\|(P w)(t, x)-\left(P w^{*}\right)(t, x)\right\|_{E} \\
\leq\left\|\int_{0}^{t} \int_{0}^{x} \frac{(t-\tau)^{r_{1}-1}(x-\xi)^{r_{2}-1}}{\Gamma\left(r_{1}\right) \Gamma\left(r_{2}\right)}\left[f\left(\tau, \xi, \bar{w}_{(\tau, \xi)}+v_{(\tau, \xi)}\right)-f\left(\tau, \xi, \bar{w}_{(\tau, \xi)}^{*}+v_{(\tau, \xi)}\right)\right] d \xi d \tau\right\|_{E} \\
\quad \quad \text { if }(t, x) \in I_{0} \\
\left\|(P w)(t, x)-\left(P w^{*}\right)(t, x)\right\|_{E} \\
\leq\left\|g_{k}\left(s_{k}, x, \bar{w}\left(s_{k}, x\right)+v\left(s_{k}, x\right)\right)-g_{k}\left(s_{k}, x, \bar{w}^{*}\left(s_{k}, x\right)+v\left(s_{k}, x\right)\right)\right\|_{E} \\
\quad+\left\|g_{k}\left(s_{k}, 0, \bar{w}\left(s_{k}, 0\right)+v\left(s_{k}, 0\right)\right)-g_{k}\left(s_{k}, 0, \bar{w}^{*}\left(s_{k}, 0\right)+v\left(s_{k}, 0\right)\right)\right\|_{E} \\
\quad+\left\|\int_{s_{k}}^{t} \int_{0}^{x} \frac{(t-\tau)^{r_{1}-1}(x-\xi)^{r_{2}-1}}{\Gamma\left(r_{1}\right) \Gamma\left(r_{2}\right)}\left[f\left(\tau, \xi, \bar{w}_{(\tau, \xi)}+v_{(\tau, \xi)}\right)-f\left(\tau, \xi, \bar{w}_{(\tau, \xi)}^{*}+v_{(\tau, \xi)}\right)\right] d \xi d \tau\right\|_{E} ; \\
\quad \text { if }(t, x) \in I_{k}, k=1, \ldots, m, \\
\left\|(P w)(t, x)-\left(P w^{*}\right)(t, x)\right\|_{E} \\
=\left\|g_{k}(t, x, \bar{w}(t, x)+v(t, x))-g_{k}\left(t, x, \bar{w}^{*}(t, x)+v(t, x)\right)\right\|_{E} ; \quad \text { if }(t, x) \in J_{k}, k=1, \ldots, m .
\end{array}\right.
$$

Thus, we get

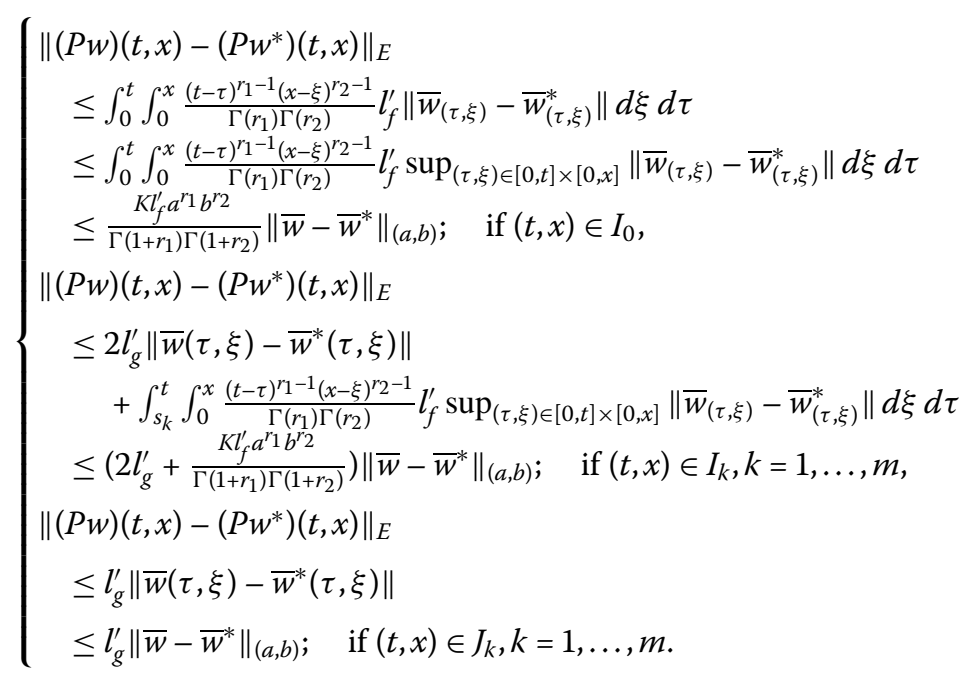

Therefore

$$
\left\|P(w)-P\left(w^{*}\right)\right\|_{(a, b)} \leq \ell^{\prime}\left\|\bar{w}-\bar{w}^{*}\right\|_{(a, b)} .
$$

By the condition (9), we conclude that $P$ is a contraction. As a consequence of Banach fixed point theorem, we deduce that $P$ has a unique fixed point $v$. Then we have

$$
\left\{\begin{aligned}
w^{*}(t, x)= & \int_{0}^{t} \int_{0}^{x} \frac{(t-\tau)^{r_{1}-1}(x-\xi)^{r_{2}-1}}{\Gamma\left(r_{1}\right) \Gamma\left(r_{2}\right)} f\left(\tau, \xi, \bar{w}_{(\tau, \xi)}^{*}+v_{(\tau, \xi)}\right) d \xi d \tau ; \quad \text { if }(t, x) \in I_{0}, \\
(P w)(t, x)= & \varphi(t)+g_{k}\left(s_{k}, x, \bar{w}^{*}\left(s_{k}, x\right)+v\left(s_{k}, x\right)\right)-g_{k}\left(s_{k}, 0, \bar{w}^{*}\left(s_{k}, 0\right)+v\left(s_{k}, 0\right)\right) \\
& +\int_{s_{k}}^{t} \int_{0}^{x} \frac{(t-\tau)^{r_{1}-1}(x-\xi)^{r_{2}-1}}{\Gamma\left(r_{1}\right) \Gamma\left(r_{2}\right)} f\left(\tau, \xi, \bar{w}_{(\tau, \xi)}^{*}+v_{(\tau, \xi)}\right) d \xi d \tau ; \\
& \text { if }(t, x) \in I_{k}, k=1, \ldots, m, \\
(P w)(t, x)= & g_{k}\left(t, x, \bar{w}^{*}(t, x)+v(t, x)\right) ; \quad \text { if }(t, x) \in J_{k}, k=1, \ldots, m .
\end{aligned}\right.
$$


Let $w \in C_{0}$ be a solution of the inequality (4). By differential this inequality, for each $(t, x) \in$ $J$, we have

$$
\left\{\begin{array}{l}
\left\|w(t, x)-\int_{0}^{t} \int_{0}^{x} \frac{(t-\tau)^{r_{1}-1}(x-\xi)^{r_{2}-1}}{\Gamma\left(r_{1}\right) \Gamma\left(r_{2}\right)} f\left(\tau, \xi, u_{(\tau, \xi)}\right) d \xi d \tau\right\|_{E} \\
\quad \leq\left\|\int_{0}^{t} \int_{0}^{x} \frac{(t-\tau)^{r_{1}-1}(x-\xi)^{r_{2}-1}}{\Gamma\left(r_{1}\right) \Gamma\left(r_{2}\right)} \Phi(\tau, \xi) d \xi d \tau\right\|_{E} ; \quad \text { if }(t, x) \in I_{0}, \\
\| u(t, x)-\varphi(t)-g_{k}\left(s_{k}, x, u\left(s_{k}, x\right)\right)+g_{k}\left(s_{k}, 0, u\left(s_{k}, 0\right)\right) \\
\quad \quad-\int_{s_{k}}^{t} \int_{0}^{x} \frac{(t-\tau)^{r_{1}-1}(x-\xi)^{r_{2}-1}}{\Gamma\left(r_{1}\right) \Gamma\left(r_{2}\right)} f\left(\tau, \xi, u_{(\tau, \xi)}\right) d \xi d \tau \|_{E} \\
\quad \leq\left\|\int_{0}^{t} \int_{0}^{x} \frac{(t-\tau)^{r_{1}-1}(x-\xi)^{r_{2}-1}}{\Gamma\left(r_{1}\right) \Gamma\left(r_{2}\right)} \Phi(\tau, \xi) d \xi d \tau\right\|_{E} ; \quad \text { if }(t, x) \in I_{k}, k=1, \ldots, m, \\
\left\|u(t, x)-g_{k}(t, x, u(t, x))\right\|_{E} \leq \Psi ; \quad \text { if }(t, x) \in J_{k}, k=1, \ldots, m .
\end{array}\right.
$$

Thus, by $\left(H_{3}\right)$ for each $(t, x) \in J$, we get

$$
\left\{\begin{array}{l}
\left\|u(t, x)-\mu(t, x)-\int_{0}^{t} \int_{0}^{x} \frac{(t-\tau)^{r_{1}-1}(x-\xi)^{r_{2}-1}}{\Gamma\left(r_{1}\right) \Gamma\left(r_{2}\right)} f\left(\tau, \xi, \bar{w}_{(\tau, \xi)}+v_{(\tau, \xi)}\right) d \xi d \tau\right\|_{E} \\
\quad \leq \lambda_{\Phi} \Phi(t, x) ; \quad \text { if }(t, x) \in I_{0}, \\
\| u(t, x)-\varphi(t)-g_{k}\left(s_{k}, x, \bar{w}\left(s_{k}, x\right)+v\left(s_{k}, x\right)\right)+g_{k}\left(s_{k}, 0, \bar{w}\left(s_{k}, 0\right)+v\left(s_{k}, 0\right)\right) \\
\quad-\int_{s_{k}}^{t} \int_{0}^{x} \frac{(t-\tau)^{r_{1}-1}(x-\xi)^{r_{2}-1}}{\Gamma\left(r_{1}\right) \Gamma\left(r_{2}\right)} f\left(\tau, \xi, \bar{w}_{(\tau, \xi)}+v_{(\tau, \xi)}\right) d \xi d \tau \|_{E} \\
\quad \leq \lambda_{\Phi} \Phi(t, x) ; \quad \text { if }(t, x) \in I_{k}, k=1, \ldots, m, \\
\left\|u(t, x)-g_{k}(t, x, \bar{w}(t, x)+v(t, x))\right\|_{E} \leq \Psi ; \quad \text { if }(t, x) \in J_{k}, k=1, \ldots, m .
\end{array}\right.
$$

Hence

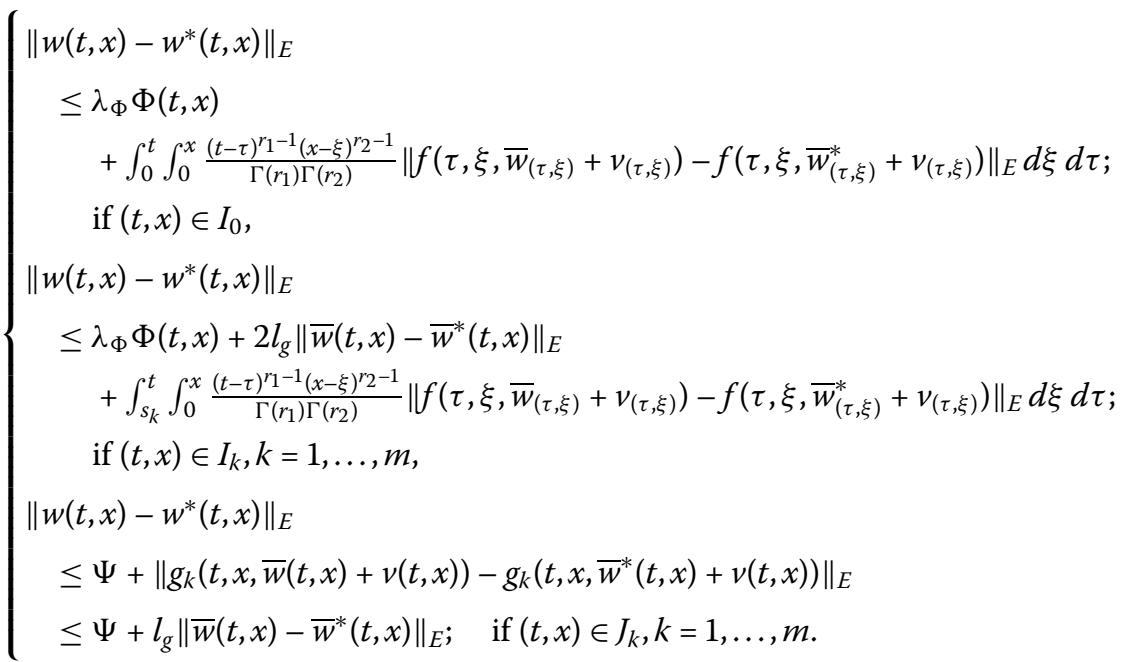

For each $(t, x) \in I_{0}$, we have

$$
\begin{aligned}
& \left\|w(t, x)-w^{*}(t, x)\right\|_{E} \\
& \leq \lambda_{\Phi} \Phi(t, x) \\
& \quad+\int_{0}^{t} \int_{0}^{x} \frac{(t-\tau)^{r_{1}-1}(x-\xi)^{r_{2}-1}}{\Gamma\left(r_{1}\right) \Gamma\left(r_{2}\right)}\left\|f\left(\tau, \xi, \bar{w}_{(\tau, \xi)}+v_{(\tau, \xi)}\right)-f\left(\tau, \xi, \bar{w}_{(\tau, \xi)}^{*}+v_{(\tau, \xi)}\right)\right\|_{E} d \xi d \tau \\
& \leq \lambda_{\Phi} \Phi(t, x)+l_{f}^{\prime} \int_{0}^{t} \int_{0}^{x} \frac{(t-\tau)^{r_{1}-1}(x-\xi)^{r_{2}-1}}{\Gamma\left(r_{1}\right) \Gamma\left(r_{2}\right)}\left\|\bar{w}_{(\tau, \xi)}-\bar{w}_{(\tau, \xi)}^{*}\right\|_{\mathcal{B}} d \xi d \tau .
\end{aligned}
$$


However,

$$
\left\|\bar{w}_{(s, t)}-\bar{w}_{(s, t)}^{*}\right\|_{\mathcal{B}} \leq K \sup _{(\tilde{s}, \tilde{t}) \in[0, s] \times[0, t]} w(\tilde{s}, \tilde{t}) .
$$

If we name $z(s, t)$ the right hand side of this inequality, then we have

$$
\left\|\bar{w}_{(s, t)}-\bar{w}_{(s, t)}^{*}\right\|_{\mathcal{B}} \leq z(t, x)
$$

and therefore, for each $(t, x) \in J$ we obtain

$$
\left\|w(t, x)-w^{*}(t, x)\right\|_{E} \leq \lambda_{\Phi} \Phi(t, x)+l_{f}^{\prime} \int_{0}^{t} \int_{0}^{x} \frac{(t-\tau)^{r_{1}-1}(x-\xi)^{r_{2}-1}}{\Gamma\left(r_{1}\right) \Gamma\left(r_{2}\right)} z(\tau, \xi) d \xi d \tau
$$

Using the above inequality and the definition of $z$, for each $(t, x) \in J$ we have

$$
z(t, x) \leq K \lambda_{\Phi} \Phi(t, x)+K l_{f}^{\prime} \int_{0}^{t} \int_{0}^{x} \frac{(t-\tau)^{r_{1}-1}(x-\xi)^{r_{2}-1}}{\Gamma\left(r_{1}\right) \Gamma\left(r_{2}\right)} z(\tau, \xi) d \xi d \tau
$$

From Lemma 2.11, there exists a constant $\delta_{1}:=\delta_{1}\left(r_{1}, r_{2}\right)$ such that

$$
\begin{aligned}
z(t, x) & \leq K \lambda_{\Phi}\left(\Phi(t, x)+K l_{f}^{\prime} \delta_{1} I_{\theta}^{r} \Phi(t, x)\right) \\
& \leq K \lambda_{\Phi}\left(1+K l_{f}^{\prime} \delta_{1} \lambda_{\Phi}\right) \Phi(t, x) \\
& :=c_{1, f, g_{k}, \Phi}^{\prime} \Phi(t, x) .
\end{aligned}
$$

Thus, for each $(t, x) \in I_{0}$, we obtain

$$
\begin{aligned}
& \left\|w(t, x)-w^{*}(t, x)\right\|_{E} \\
& \quad \leq \lambda_{\Phi} \Phi(t, x)+l_{f}^{\prime} \int_{0}^{t} \int_{0}^{x} \frac{(t-\tau)^{r_{1}-1}(x-\xi)^{r_{2}-1}}{\Gamma\left(r_{1}\right) \Gamma\left(r_{2}\right)} c_{1, f, g_{k}, \Phi}^{\prime} \Phi(\tau, \xi) d \xi d \tau \\
& \quad \leq\left(\lambda_{\Phi}+l_{f}^{\prime} c_{1, f, g_{k}, \Phi}^{\prime} \lambda_{\Phi}\right) \Phi(t, x) \\
& \quad:=c_{1, f, g_{k}, \Phi} \Phi(t, x) .
\end{aligned}
$$

Hence, for each $(t, x) \in I_{0}$, we get

$$
\|u(t, x)-v(t, x)\|_{E} \leq c_{1, f, g_{k}, \Phi}(\Psi+\Phi(t, x))
$$

Now, for each $(t, x) \in I_{k}, k=1, \ldots, m$, we have

$$
\begin{aligned}
& \left\|w(t, x)-w^{*}(t, x)\right\|_{E} \\
& \leq \lambda_{\Phi} \Phi(t, x)+2 l_{g}^{\prime}\left\|\bar{w}(t, x)-\bar{w}^{*}(t, x)\right\|_{E} \\
& \quad+l_{f} \int_{s_{k}}^{t} \int_{0}^{x} \frac{(t-\tau)^{r_{1}-1}(x-\xi)^{r_{2}-1}}{\Gamma\left(r_{1}\right) \Gamma\left(r_{2}\right)}\left\|\bar{w}_{(\tau, \xi)}-\bar{w}_{(\tau, \xi)}^{*}\right\|_{\mathcal{B}} d \xi d \tau .
\end{aligned}
$$


Then we obtain

$$
\begin{aligned}
& \left\|w(t, x)-w^{*}(t, x)\right\|_{E} \\
& \quad \leq \frac{\lambda_{\Phi}}{1-2 l_{g}^{\prime}} \Phi(t, x)+\frac{l_{f}^{\prime}}{1-2 l_{g}^{\prime}} \int_{s_{k}}^{t} \int_{0}^{x} \frac{(t-\tau)^{r_{1}-1}(x-\xi)^{r_{2}-1}}{\Gamma\left(r_{1}\right) \Gamma\left(r_{2}\right)}\left\|\bar{w}_{(\tau, \xi)}-\bar{w}_{(\tau, \xi)}^{*}\right\|_{\mathcal{B}} d \xi d \tau .
\end{aligned}
$$

Again, from Lemma 2.11, there exists a constant $\delta_{2}:=\delta_{2}\left(r_{1}, r_{2}\right)$ such that

$$
\begin{aligned}
z(t, x) & \leq \frac{K \lambda_{\Phi}}{1-2 l_{g}^{\prime}}\left(\Phi(t, x)+\frac{K l_{f}^{\prime} \delta_{2}}{1-2 l_{g}^{\prime}} I_{\theta_{k}}^{r} \Phi(t, x)\right) \\
& \leq \frac{K \lambda_{\Phi}}{1-2 l_{g}}\left(1+\frac{K l_{f}^{\prime} \delta_{2} \lambda_{\Phi}}{1-2 l_{g}}\right) \Phi(t, x) \\
& :=c_{2, f, g_{k}, \Phi}^{\prime} \Phi(t, x),
\end{aligned}
$$

and, then

$$
\begin{aligned}
& \left\|w(t, x)-w^{*}(t, x)\right\|_{E} \\
& \quad \leq \frac{\lambda_{\Phi}}{1-2 l_{g}^{\prime}} \Phi(t, x)+\frac{l_{f}^{\prime}}{1-2 l_{g}^{\prime}} \int_{s_{k}}^{t} \int_{0}^{x} \frac{(t-\tau)^{r_{1}-1}(x-\xi)^{r_{2}-1}}{\Gamma\left(r_{1}\right) \Gamma\left(r_{2}\right)} c_{2, f, g_{k}, \Phi}^{\prime} \Phi(\tau, \xi) d \xi d \tau \\
& \quad \leq \frac{\lambda_{\Phi}+l_{f}^{\prime} \lambda_{\Phi} c_{2, f, g_{k}, \Phi}^{\prime}}{1-2 l_{g}^{\prime}} \Phi(t, x) \\
& \quad:=c_{2, f, g_{k}, \Phi} \Phi(t, x) .
\end{aligned}
$$

Hence, for each $(t, x) \in I_{k}, k=1, \ldots, m$, we get

$$
\|u(t, x)-v(t, x)\|_{E} \leq c_{2, f, g_{k}, \Phi}(\Psi+\Phi(t, x))
$$

Now, for each $(t, x) \in J_{k}, k=1, \ldots, m$, we have

$$
\left\|w(t, x)-w^{*}(t, x)\right\|_{E} \leq \Psi+l_{g}^{\prime}\left\|\bar{w}(t, x)-\bar{w}^{*}(t, x)\right\|_{E}
$$

This gives

$$
\|u(t, x)-v(t, x)\|_{E} \leq \frac{\Psi}{1-l_{g}}:=c_{3, f, g_{k}, \Phi} \Psi .
$$

Thus, for each $(t, x) \in J_{k}, k=1, \ldots, m$, we get

$$
\|u(t, x)-v(t, x)\|_{E} \leq c_{3, f, g_{k}, \Phi}(\Psi+\Phi(t, x)) .
$$

Set $c_{f, g_{k}, \Phi}:=\max _{i \in\{1,2,3\}} c_{i, f, g_{k}, \Phi}$. Hence, for each $(t, x) \in J$, we obtain

$$
\|u(t, x)-v(t, x)\|_{E} \leq c_{f, g_{k}, \Phi}(\Psi+\Phi(t, x)) .
$$

Consequently, problem (2) is generalized Ulam-Hyers-Rassias stable. 


\section{Examples}

Example 6.1 Let $E=l^{1}=\left\{w=\left(w_{1}, w_{2}, \ldots, w_{n}, \ldots\right): \sum_{n=1}^{\infty}\left|w_{n}\right|<\infty\right\}$, be the Banach space with norm

$$
\|w\|_{E}=\sum_{n=1}^{\infty}\left|w_{n}\right|
$$

Consider partial fractional differential equations with noninstantaneous impulses and finite delay of the form

$$
\left\{\begin{array}{l}
{ }^{c} D_{\theta_{k}}^{r} u(t, x)=f(t, x, u(t-1, x-2)) ; \quad \text { if }(t, x) \in([0,1] \cup(2,3]) \times[0,1], k \in\{0,1\} \\
u(t, x)=g(t, x, u(t, x)) ; \quad \text { if }(t, x) \in(1,2] \times[0,1] \\
u(t, x)=1+x^{2}+e^{t} ; \quad(t, x) \in[-1,3] \times[-2,1] \backslash(0,3] \times(0,1] \\
u(t, 0)=1+e^{t} ; \quad t \in[0,3] \\
u(0, x)=2+x^{2} ; \quad x \in[0,1]
\end{array}\right.
$$

where $r=\left(r_{1}, r_{2}\right) \in(0,1] \times(0,1], \theta_{0}=(0,0), \theta_{1}=(2,0), \alpha=-1, \beta=-2,0=s_{0}<t_{1}=1<s_{1}=$ $2<t_{2}=3, u=\left(u_{1}, u_{2}, \ldots, u_{n}, \ldots\right), f=\left(f_{1}, f_{2}, \ldots, f_{n}, \ldots\right), g=\left(g_{1}, g_{2}, \ldots, g_{n}, \ldots\right)$,

$$
\begin{aligned}
& { }^{c} D_{\theta}^{r} u=\left({ }^{c} D_{\theta}^{r} u_{1},{ }^{c} D_{\theta}^{r} u_{2}, \ldots,{ }^{c} D_{\theta}^{r} u_{n}, \ldots\right), \\
& f_{n}\left(t, x, u_{n}\right)=\frac{1}{\left(1+110 e^{t+x}\right)\left(1+\left\|u_{n}\right\|_{\mathcal{C}}\right)} ; \quad(t, x) \in([0,1] \cup(2,3]) \times[0,1] \text { and } n \in \mathbb{N},
\end{aligned}
$$

$\mathcal{C}:=C_{(1,2)}$ and

$$
g_{n}\left(t, x, u_{n}\right)=\frac{1}{1+110 e^{t+x}} \arctan \left(t^{2}+x^{2}+\left|u_{n}\right|\right) ; \quad(t, x) \in(1,2] \times[0,1] \text { and } n \in \mathbb{N} .
$$

Clearly, the functions $f$ and $g$ are continuous. For each $n \in \mathbb{N}, u, \bar{u} \in E$ and $(t, x) \in([0,1] \cup$ $(2,3]) \times[0,1]$, we have

$$
\left|f_{n}\left(t, x, u_{n(t, x)}\right)-f_{n}\left(t, x, \bar{u}_{n(t, x)}\right)\right| \leq \frac{1}{111}\left\|u_{n}-\bar{u}_{n}\right\|_{\mathcal{C}} .
$$

Thus, for each $u, \bar{u} \in E$ and $(t, x) \in([0,1] \cup(2,3]) \times[0,1]$ we get

$$
\begin{aligned}
\left\|f\left(t, x, u_{(t, x)}\right)-f\left(t, x, \bar{u}_{(t, x)}\right)\right\|_{E} & =\sum_{n=1}^{\infty}\left|f_{n}\left(t, x, u_{(t, x)}\right)-f_{n}\left(t, x, \bar{u}_{(t, x)}\right)\right| \\
& \leq \frac{1}{111} \sum_{n=1}^{\infty}\left\|u_{n}-\bar{u}_{n}\right\|_{\mathcal{C}} \\
& =\frac{1}{111}\left\|u_{n}-\bar{u}_{n}\right\|_{\mathcal{C}} .
\end{aligned}
$$

Also, for each $n \in \mathbb{N}, u, \bar{u} \in E$ and $(t, x) \in(1,2] \times[0,1]$, we get

$$
\|g(t, x, u(t, x))-g(t, x, \bar{u}(t, x))\|_{E} \leq \frac{1}{111}\|u-\bar{u}\|_{E} .
$$


Hence the conditions $\left(H_{1}\right)$ and $\left(H_{2}\right)$ are satisfied with $l_{f}=l_{g}=\frac{1}{111}$. We shall show that condition (8) holds with $a=3$ and $b=1$. Indeed, for each $\left(r_{1}, r_{2}\right) \in(0,1] \times(0,1]$ we get

$$
\begin{aligned}
\ell & =2 l_{g}+\frac{l_{f} a^{r_{1}} b^{r_{2}}}{\Gamma\left(1+r_{1}\right) \Gamma\left(1+r_{2}\right)} \\
& =\frac{2}{111}+\frac{3^{r_{1}}}{111 \Gamma\left(1+r_{1}\right) \Gamma\left(1+r_{2}\right)} \\
& <\frac{14}{111}<1 .
\end{aligned}
$$

By Theorem 3.4, the problem (10) has a unique solution defined on $[-1,3] \times[-2,1]$. Finally, the hypothesis $\left(H_{3}\right)$ is satisfied with $\Phi(t, x)=t x^{2}$ and $\lambda_{\Phi}=\frac{2 \times 3 r_{1}}{\Gamma\left(2+r_{1}\right) \Gamma\left(3+r_{2}\right)}$. Indeed, for each $(t, x) \in[0,3] \times[0,1]$ we get

$$
\left(I_{\theta}^{r} \Phi\right)(t, x)=\frac{\Gamma(2) \Gamma(3) t^{1+r_{1}} x^{2+r_{2}}}{\Gamma\left(2+r_{1}\right) \Gamma\left(3+r_{2}\right)} \leq \frac{2 \times 3^{r_{1}} t x^{2}}{\Gamma\left(2+r_{1}\right) \Gamma\left(3+r_{2}\right)}=\lambda_{\Phi} \Phi(t, x) .
$$

Consequently, Theorem 3.4 implies that the problem (10) is generalized Ulam-HyersdzRassias stable.

Example 6.2 Consider now partial differential equations with noninstantaneous impulses and infinite delay of the form

$$
\left\{\begin{array}{l}
{ }^{c} D_{\theta_{k}}^{r} u(t, x)=f\left(t, x, u_{(t, x)}\right) ; \quad \text { if }(t, x) \in([0,1] \cup(2,3]) \times[0,1], k \in\{0,1\} \\
u(t, x)=g(t, x, u(t, x)) ; \quad \text { if }(t, x) \in(1,2] \times[0,1] \\
u(t, x)=t+x^{2} ; \quad \text { if }(t, x) \in \tilde{J}:=[-\infty, 3] \times[-\infty, 1] \backslash(0,3] \times(0,1] \\
u(t, 0)=t ; \quad t \in[0,3], \\
u(0, x)=x^{2} ; \quad x \in[0,1] \\
\varphi(0)=\psi(0),
\end{array}\right.
$$

where

$$
\begin{aligned}
& r=\left(r_{1}, r_{2}\right) \in(0,1] \times(0,1], \quad \theta_{0}=(0,0), \quad \theta_{1}=(2,0), \\
& 0=s_{0}<t_{1}=1<s_{1}=2<t_{2}=3, \\
& f\left(t, x, u_{(t, x)}\right)=\frac{c e^{t+x-\gamma(t+x)}\left\|u_{(t, x)}\right\|}{\left(e^{t+x}+e^{-t-x}\right)\left(1+\| u_{(t, x)}\right) \|} ; \quad \text { if }(t, x) \in([0,1] \cup(2,3]) \times[0,1], \\
& c=\frac{11 \times 3^{r_{1}}}{\Gamma\left(1+r_{1}\right) \Gamma\left(1+r_{2}\right)}, \gamma>0 \text { and } \\
& g(t, x, u)=\frac{1}{1+10 e^{t+x}} \arctan \left(t^{2}+x^{2}+|u|\right) ; \quad(t, x) \in(1,2] \times[0,1] .
\end{aligned}
$$

Let

$$
B_{\gamma}=\left\{u \in C((-\infty, 0] \times(-\infty, 0], \mathbb{R}): \lim _{\|(\theta, \eta)\| \rightarrow \infty} e^{\gamma(\theta+\eta)} u(\theta, \eta) \text { exists in } \mathbb{R}\right\}
$$


The norm of $B_{\gamma}$ is given by

$$
\|u\|_{\gamma}=\sup _{(\theta, \eta) \in(-\infty, 0] \times(-\infty, 0]} e^{\gamma(\theta+\eta)}|u(\theta, \eta)| .
$$

Let

$$
\mathcal{E}:=[0,1] \times\{0\} \cup\{0\} \times[0,1],
$$

and $u:(-\infty, 3] \times(-\infty, 1] \rightarrow \mathbb{R}$ such that $u_{(t, x)} \in B_{\gamma}$ for $(t, x) \in \mathcal{E}$, then

$$
\begin{aligned}
\lim _{\|(\theta, \eta)\| \rightarrow \infty} e^{\gamma(\theta+\eta)} u_{(t, x)}(\theta, \eta) & =\lim _{\|(\theta, \eta)\| \rightarrow \infty} e^{\gamma(\theta-x+\eta-y)} u(\theta, \eta) \\
& =e^{\gamma(x+y)} \lim _{\|(\theta, \eta)\| \rightarrow \infty} u(\theta, \eta)<\infty .
\end{aligned}
$$

Hence $u_{(t, x)} \in B_{\gamma}$. Finally we prove that

$$
\left\|u_{(t, x)}\right\|_{\gamma}=K \sup \{|u(\tau, \xi)|:(\tau, \xi) \in[0, t] \times[0, x]\}+M \sup \left\{\left\|u_{(\tau, \xi)}\right\|_{\gamma}:(\tau, \xi) \in \mathcal{E}_{(t, x)}\right\}
$$

where $K=M=1$ and $H=1$. If $t+\theta \leq 0, x+\eta \leq 0$ we get

$$
\left\|u_{(t, x)}\right\|_{\gamma}=\sup \{|u(\tau, \xi)|:(\tau, \xi) \in(-\infty, 0] \times(-\infty, 0]\},
$$

and if $t+\theta \geq 0, x+\eta \geq 0$ then we have

$$
\left\|u_{(t, x)}\right\|_{\gamma}=\sup _{(\tau, \xi) \in[0, t] \times[0, x]}|u(\tau, \xi)| .
$$

Thus for all $(t+\theta, x+\eta) \in[0,3] \times[0,1]$, we get

$$
\left\|u_{(t, x)}\right\|_{\gamma}=\sup _{(\tau, \xi) \in(-\infty, 0] \times(-\infty, 0]}|u(\tau, \xi)|+\sup _{(\tau, \xi) \in[0, t] \times[0, x]}|u(\tau, \xi)| .
$$

Then

$$
\left\|u_{(t, x)}\right\|_{\gamma}=\sup _{(s, t) \in E}\left\|u_{(s, t)}\right\|_{\gamma}+\sup _{(s, t) \in[0, t] \times[0, x]}|u(s, t)| .
$$

$\left(B_{\gamma},\|\cdot\|_{\gamma}\right)$ is a Banach space. We conclude that $B_{\gamma}$ is a phase space.

For each $u, \bar{u} \in B_{\gamma}$ and $(t, x) \in([0,1] \cup(2,3]) \times[0,1]$, we have

$$
\begin{aligned}
\left|f\left(t, x, u_{(t, x)}\right)-f\left(t, x, \bar{u}_{(t, x)}\right)\right| & \leq \frac{e^{t+x}\|u-\bar{u}\|_{B}}{c\left(e^{t+x}+e^{-t-x}\right)} \\
& \leq \frac{1}{c}\|u-\bar{u}\|_{B} .
\end{aligned}
$$

Hence condition $\left(H_{1}\right)$ is satisfied with $l_{f}=\frac{1}{c}$. Also, for each $u, \bar{u} \in \mathbb{R}$ and $(t, x) \in(1,2] \times[0,1]$, we have we get

$$
|g(t, x, u(t, x))-g(t, x, \bar{u}(t, x))| \leq \frac{1}{11}|u-\bar{u}|
$$


Hence the condition $\left(H_{2}\right)$ is satisfied with $l_{g}=\frac{1}{11}$. We shall show that condition (9) holds with $a=3$ and $b=K=1$. Indeed,

$$
\begin{aligned}
\ell^{\prime} & =2 l_{g}^{\prime}+\frac{K l_{f}^{\prime} a^{r_{1}} b^{r_{2}}}{\Gamma\left(1+r_{1}\right) \Gamma\left(1+r_{2}\right)} \\
& =\frac{2}{11}+\frac{3^{r_{1}}}{c \Gamma\left(1+r_{1}\right) \Gamma\left(1+r_{2}\right)} \\
& \leq \frac{2}{11}+\frac{1}{11}=\frac{3}{11}<1 .
\end{aligned}
$$

By Theorem 5.1, the problem (11) has a unique solution defined on $(-\infty, 3] \times(-\infty, 1]$. Moreover, the hypothesis $\left(H_{3}\right)$ is satisfied with $\Phi(t, x)=t x^{2}$ and $\lambda_{\Phi}=\frac{2 \times 3^{r_{1}}}{\Gamma\left(2+r_{1}\right) \Gamma\left(3+r_{2}\right)}$. Indeed, for each $(t, x) \in[0,3] \times[0,1]$ we get

$$
\left(I_{\theta}^{r} \Phi\right)(t, x)=\frac{\Gamma(2) \Gamma(3) t^{1+r_{1}} x^{2+r_{2}}}{\Gamma\left(2+r_{1}\right) \Gamma\left(3+r_{2}\right)} \leq \frac{2 \times 3^{r_{1}} t x^{2}}{\Gamma\left(2+r_{1}\right) \Gamma\left(3+r_{2}\right)}=\lambda_{\Phi} \Phi(t, x) .
$$

Consequently, Theorem 5.1 implies that the problem (11) is generalized Ulam-HyersRassias stable.

Competing interests

The authors declare that they have no competing interests.

\title{
Authors' contributions
}

SA and MB contributed to Sections 1, 2,3, and 5. AA and YZ contributed to Sections 4 and 6 .

\begin{abstract}
Author details
'Laboratory of Mathematics, University of Saïda, P.O. Box 138, Saïda, 20000, Algeria. ${ }^{2}$ Laboratory of Mathematics, University of Sidi Bel-Abbes, P.O. Box 89, Sidi Bel-Abbès, 22000, Algeria. ${ }^{3}$ Nonlinear Analysis and Applied Mathematics (NAAM) Research Group, Faculty of Science, King Abdulaziz University, P.O. Box 80203, Jeddah, 21589, Saudi Arabia. ${ }^{4}$ Faculty of Mathematics and Computational Science, Xiangtan University, Hunan, 411105, P.R. China.
\end{abstract}

\section{Acknowledgements}

The authors would like to thank all the anonymous reviewers and the editors for their helpful advice and hard work. The work was supported by the National Natural Science Foundation of China (No. 11671339).

Received: 20 November 2016 Accepted: 7 February 2017 Published online: 09 March 2017

\section{References}

1. Abbas, S, Benchohra, M, N'Guérékata, GM: Topics in Fractional Differential Equations. Springer, New York (2012)

2. Kilbas, AA, Srivastava, HM, Trujillo, JJ: Theory and Applications of Fractional Differential Equations. Elsevier Science B.V., Amsterdam (2006)

3. Miller, KS, Ross, B: An Introduction to the Fractional Calculus and Differential Equations. Wiley, New York (1993)

4. Zhou, Y: Basic Theory of Fractional Differential Equations. World Scientific, Singapore (2014)

5. Zhou, Y: Fractional Evolution Equations and Inclusions: Analysis and Control. Academic Press, San Diego (2016)

6. Abbas, S, Benchohra, M: Impulsive partial functional integro-differential equations of fractional order. Commun. Appl. Anal. 16(2), 249-260 (2012)

7. Abbas, S, Benchohra, M: Existence and stability for partial functional integro-differential equations of fractional order with multiple time delay. Nonlinear Stud. 20(2), 149-162 (2013)

8. Abbas, S, Benchohra, M: Ulam-Hyers stability for the Darboux problem for partial fractional differential and integro-differential equations via Picard operators. Results Math. 65(1-2), 67-79 (2014)

9. Abbas, S, Benchohra, M, Nieto, JJ: Ulam stabilities for impulsive partial fractional differential equations. Acta Univ. Palacki. Olomuc., Fac. Rerum Nat., Math. 53, 5-17 (2014)

10. Abbas, S, Benchohra, M, Vityuk, AN: On fractional order derivatives and Darboux problem for implicit differential equations. Fract. Calc. Appl. Anal. 15(2), 168-182 (2012)

11. Abbas, S, Benchohra, M, Zhou, Y: Fractional order partial functional differential inclusions with infinite delay. Proc. A. Razmadze Math. Inst. 154, 1-19 (2010)

12. Ahmad, B, Alsaedi, A: Existence and uniqueness of solutions for coupled systems of higher-order nonlinear fractional differential equations. Fixed Point Theory Appl. 2010, 364560 (2010)

13. Ahmad, B, Nieto, JJ, Alsaedi, A: A study of nonlinear Langevin equation involving two fractional orders in different intervals. Nonlinear Anal., Real World Appl. 13, 599-606 (2012)

14. Diethelm, K, Ford, NJ: Analysis of fractional differential equations. J. Math. Anal. Appl. 265, 229-248 (2002) 
15. Kilbas, AA, Marzan, SA: Nonlinear differential equations with the Caputo fractional derivative in the space of continuously differentiable functions. Differ. Equ. 41, 84-89 (2005)

16. Podlubny, I, Petraš, I, Vinagre, BM, O'Leary, P, Dorčak, L: Analogue realizations of fractional-order controllers. Nonlinear Dyn. 29, 281-296 (2002)

17. Vityuk, AN, Golushkov, AV: Existence of solutions of systems of partial differential equations of fractional order Nonlinear Oscil. 7(3), 318-325 (2004)

18. Yang, XJ, Tenreiro Machado, JA, Baleanu, D, Cattani, C: On exact traveling-wave solutions for local fractional Korteweg-de Vries equation. Chaos, Interdiscip. J. Nonlinear Sci. 26(8), 084312 (2016)

19. Yang, XJ, Srivastava, HM, Cattani, C: Local fractional homotopy perturbation method for solving fractal partial differential equations arising in mathematical physics. Rom. Rep. Phys. 67(3), 752-761 (2015)

20. Yang, XJ, Srivastava, HM: An asymptotic perturbation solution for a linear oscillator of free damped vibrations in fractal medium described by local fractional derivatives. Commun. Nonlinear Sci. Numer. Simul. 29(1), 499-504 (2015)

21. Yang, XJ, Srivastava, HM, Machado, JA: A new fractional derivative without singular kernel: application to the modelling of the steady heat flow. Therm. Sci. 20(2), 753-756 (2016)

22. Zhou, Y, Vijayakumar, V, Murugesu, R: Controllability for fractional evolution inclusions without compactness. Evol. Equ. Control Theory 4, 507-524 (2015)

23. Zhou, Y, Peng, L: On the time-fractional Navier-Stokes equations. Comput. Math. Appl. 73(6), 874-891 (2017). doi:10.1016/j.camwa.2016.03.026

24. Zhou, Y, Peng, L: Weak solutions of the time-fractional Navier-Stokes equations and optimal control. Comput. Math. Appl. 73(6), 1016-1027 (2017). doi:10.1016/j.camwa.2016.07.007

25. Zhou, Y, Zhang, L: Existence and multiplicity results of homoclinic solutions for fractional Hamiltonian systems. Comput. Math. Appl. 73(6), 1325-1345 (2017). doi:10.1016/j.camwa.2016.04.041

26. Wang, JR, Fečkan, M, Zhou, Y: On the stability of first order impulsive evolution equations. Opusc. Math. 34, 639-657 (2014)

27. Hernández, E, O'Regan, D: On a new class of abstract impulsive differential equations. Proc. Am. Math. Soc. 141, 1641-1649 (2013)

28. Pierri, M, O'Regan, D, Rolnik, V: Existence of solutions for semi-linear abstract differential equations with not instantaneous. Appl. Math. Comput. 219, 6743-6749 (2013)

29. Rus, IA: Ulam stabilities of ordinary differential equations in a Banach space. Carpath. J. Math. 26, 103-107 (2010)

30. Wang, JR, Fečkan, M, Zhou, Y: Ulam's type stability of impulsive ordinary differential equations. J. Math. Anal. Appl. 395, 258-264 (2012)

31. Henry, D: Geometric Theory of Semilinear Parabolic Partial Differential Equations. Springer, Berlin (1989)

32. Hale, J, Kato, J: Phase space for retarded equations with infinite delay. Funkc. Ekvacioj 21, 11-41 (1978)

33. Czlapinski, T: On the Darboux problem for partial differential-functional equations with infinite delay at derivatives. Nonlinear Anal. 44, 389-398 (2001)

34. Czlapinski, T: Existence of solutions of the Darboux problem for partial differential-functional equations with infinite delay in a Banach space. Comment. Math. Prace Mat. 35, 111-122 (1995)

\section{Submit your manuscript to a SpringerOpen ${ }^{\ominus}$ journal and benefit from:}

$\checkmark$ Convenient online submission

- Rigorous peer review

- Immediate publication on acceptance

- Open access: articles freely available online

- High visibility within the field

- Retaining the copyright to your article 